\title{
MPCA: Multilinear Principal Component Analysis of Tensor Objects
}

\author{
Haiping Lu, K.N. Plataniotis and A.N. Venetsanopoulos \\ The Edward S. Rogers Sr. Department of Electrical and Computer Engineering \\ University of Toronto, M5S 3G4, Canada \\ Email: hplu@ieee.org
}

\begin{abstract}
This paper introduces a multilinear principal component analysis (MPCA) framework for tensor object feature extraction. Objects of interest in many computer vision and pattern recognition applications, such as $2 \mathrm{D} / 3 \mathrm{D}$ images and video sequences are naturally described as tensors or multilinear arrays. The proposed framework performs feature extraction by determining a multilinear projection that captures most of the original tensorial input variation. The solution is iterative in nature and it proceeds by decomposing the original problem to a series of multiple projection subproblems. As part of this work, methods for subspace dimensionality determination are proposed and analyzed. It is shown that the MPCA framework discussed in this work supplants existing heterogeneous solutions such as the classical principal component analysis (PCA) and its two-dimensional (2DPCA) variant. Finally, a tensor object recognition system is proposed with the introduction of a discriminative tensor feature selection mechanism and a novel classification strategy, and applied to the problem of gait recognition. Results presented here indicate MPCA's utility as a feature extraction tool. It is shown that even without a fully optimized design, an MPCA-based gait recognition module achieves highly competitive performance and compares favorably to the state-of-the-art gait recognizers.
\end{abstract}

Index Terms

Multilinear principal component analysis (MPCA), tensor objects, dimensionality reduction, feature extraction, gait recognition.

This paper was presented in part at the Biometrics Consortium/IEEE 2006 Biometrics Symposium, Baltimore, Maryland, September 19-21, 2006. 


\section{INTRODUCTION}

The term tensor object is used here to denote a multi-dimensional object, the elements of which are to be addressed by more than two indices [1]. The number of indices used in the description defines the order of the tensor object and each index defines one of the so-called "modes". Many image and video data are naturally tensor objects. For example, color images are three-dimensional (3rd-order tensor) objects with column, row and color modes [2]. Gait silhouette sequences, the input to most if not all gait recognition algorithms [3]-[7], as well as other gray-scale video sequences can be viewed as 3rdorder tensors with column, row and time modes. Naturally, color video sequences are 4th-order tensors with the addition of a color mode. In the most active area of biometrics research, namely that of face recognition, three-dimensional face detection and recognition using 3D information with column, row and depth modes, in other words a 3rd-order tensor, has emerged as an important research direction [8]-[10]. Moreover, the research problem of matching still probe images to surveillance video sequences can be viewed as a pattern recognition problem in a 3rd-order tensorial setting [11]. Beyond biometrics signal analysis, many other computer vision and pattern recognition tasks can be also viewed as problems in a multilinear domain. Such tasks include 3D object recognition tasks [12] in machine vision, medical image analysis and content-based retrieval, space-time analysis of video sequences for gesture recognition [13] and activity recognition [14] in human-computer interaction (HCI), and space-time super-resolution [15] for digital cameras with limited spatial and temporal resolution. The wide range of applications explains the authors' belief that a comprehensive study of a specialized feature extraction problem, such as multilinear feature extraction, is worthwhile.

A typical tensor object in pattern recognition or machine vision applications is commonly specified in a high-dimensional tensor space. Recognition methods operating directly on this space suffer from the socalled curse of dimensionality [16]: handling high-dimensional samples is computationally expensive and many classifiers perform poorly in high-dimensional spaces given a small number of training samples. However, since the entries of a tensor object are often highly correlated with surrounding entries, it is reasonable to assume that the tensor objects encountered in most applications of interest are highly constrained and thus the tensors are confined to a subspace, a manifold of intrinsically low dimension [16], [17]. Feature extraction or dimensionality reduction is thus an attempt to transform a high-dimensional data set into a low-dimensional equivalent representation while retaining most of the information regarding the underlying structure or the actual physical phenomenon [18].

Principal Component Analysis (PCA) is a well-known unsupervised linear technique for dimensionality 
reduction. The central idea behind PCA is to reduce the dimensionality of a data set consisting of a larger number of interrelated variables, while retaining as much as possible the variation present in the original data set [19]. This is achieved by transforming to a new set of variables, the so-called principal components (PCs), which are uncorrelated, and ordered so that the first few retain most of the original data variation. Naive application of PCA to tensor objects requires their reshaping into vectors with high dimensionality (vectorization), which obviously results in high processing cost in terms of increased computational and memory demands. For example, vectorizing a typical gait silhouette sequence of size $(120 \times 80 \times 20)$ results in a vector with dimensionality $(192,000 \times 1)$, the singular value decomposition (SVD) or eigen-decomposition processing of which may be beyond the computing processing capabilities of many computing devices. Beyond implementation issues, it is well understood that reshaping breaks the natural structure and correlation in the original data, removing redundancies and/or higher order dependencies present in the original data set and losing potentially more compact or useful representations that can be obtained in the original form [20]. Vectorization as PCA preprocessing ignores the fact that tensor objects are naturally multi-dimensional objects, e.g., gait sequences are three-dimensional objects, instead of one-dimensional objects. Therefore, a dimensionality reduction algorithm operating directly on a tensor object rather than its vectorized version is desirable.

Recently, dimensionality reduction solutions representing images as matrices (second order tensors) rather than vectors (first order tensors) have been introduced. A two-dimensional PCA (2DPCA) algorithm is proposed in [21], where the image covariance matrix is constructed using image matrices as inputs. However, a linear transformation is applied only to the right side of the input image matrices. As a result, image data is projected in one mode only, resulting in poor dimensionality reduction. The less restrictive 2DPCA algorithm introduced in [20] takes into account the spatial correlation of the image pixels within a localized neighborhood. Two linear transforms are applied to both the left and the right side of the input image matrices. Thus, projections in both modes are calculated and better dimensionality reduction results are obtained according to [22]. Similarly to the solutions introduced in [21] and [22], the so-called Tensor Subspace Analysis algorithm of [23] represents the input image as a matrix residing in a tensor space and attempts to detect local geometrical structure in that tensor space by learning a lower dimensional tensor subspace.

For the theoretically inclined reader, it should be noted that there are some recent developments in the analysis of higher-order tensors. The higher-order singular value decomposition (HOSVD) solution, which extends SVD to higher-order tensors, was formulated in [24] and its computation leads to the calculation of $N$ (the order) different matrix SVDs of unfolded matrices. An alternating-least-square (ALS) algorithm 
for the best Rank- $\left(R_{1}, R_{2}, \ldots, R_{N}\right)$ approximation of higher-order tensors was studied in [1], where tensor data was projected into a lower dimensional tensor space iteratively. The application of HOSVD truncation and the best Rank- $\left(R_{1}, R_{2}, \ldots, R_{N}\right)$ approximation to dimensionality reduction in independent component analysis (ICA) was discussed in [25]. These multilinear algorithms have been used routinely for multiple factor analysis [26], [27], where input data such as images are still represented as vectors but with these vectors arranged into a tensor for the subsequent analysis of the multiple factors involved in image/video formation. It should be added that in [25]-[27], the tensor data under consideration is projected in the original coordinate without data centering. However, for classification/recognition applications where eigenproblem solutions are attempted, the eigen-decomposition in each mode can be influenced by the mean (average) of the data set.

Recently, there have been several attempts to develop multilinear subspace algorithms for tensor object feature extraction and classification. In [28], a heuristic MPCA approach based on HOSVD was proposed. The MPCA formulation in [29] targets optimal reconstruction applications (where data is not centered) with a solution built in a manner similar to that of [1]. It should be noted that the solution in [29] was focused on reconstruction not recognition and that it did not cover a number of important algorithmic issues, namely: initialization, termination, convergence and subspace dimensionality determination. When applied to the problem of tensor object recognition, the methodology described in [29] uses all the entries in the projected tensor for recognition although the discrimination power of these entries varies considerably. There is also a recent work on multilinear discriminant analysis (MLDA) [30], [31], named Discriminant Analysis with Tensor Representation (DATER), where an iterative algorithm similar to ALS of [1] is utilized in order to maximize a tensor-based discriminant criterion. Unfortunately, this MLDA variant does not converge and it appears to be extremely sensitive to parameter settings [32]. As the number of possible subspace dimensions for tensor objects is extremely high (e.g., there are 225280 possible subspace dimensions for the gait recognition problem considered in this work), exhaustive testing for determination of parameters is not feasible. Consequently, the algorithmic solution of [30], [31] cannot be used to effectively determine subspace dimensionality in a comprehensive and systematic manner.

Motivated by the works briefly reviewed here, this paper introduces a new multilinear principal component analysis (MPCA) formulation for tensor object dimensionality reduction and feature extraction. The proposed solution follows the classical PCA paradigm. Operating directly on the original tensorial data, the proposed MPCA is a multilinear algorithm performing dimensionality reduction in all tensor modes seeking those bases in each mode that allow projected tensors to capture most of the variation present in the original tensors. 
The main contributions of this work include:

1) The introduction of a new MPCA framework for tensor object dimensionality reduction and feature extraction using tensor representation. The framework is introduced from the perspective of capturing the original tensors' variation. It provides a systematic procedure to determine effective representations of tensor objects. This contrasts to previous work such as those reported in [16], [26], [27], where vector, not tensor, representation was used, and the works reported in [20], [21], [23], where matrix representation was utilized. It also differs from the works reported in [1], [24], [25], where tensor data are processed as part of a reconstruction/regression solution. Furthermore, unlike previous attempts, such as the one in [29], design issues of paramount importance in practical applications, such as the initialization, termination, convergence of the algorithm and the determination of the subspace dimensionality, are discussed in detail.

2) The definition of eigentensors and $n$-mode eigenvalues as counterparts of the eigenvectors and eigenvalues in classical PCA. The geometrical interpretation of these concepts is provided, enabling a deeper understanding of the main principles and facilitating the application of multilinear feature extraction.

3) The presentation of a recognition system that selects discriminative tensor features from tensor objects and uses a novel weighting method for classification. This differs from traditional vectorbased object recognition systems [16] that often encounter computational and memory difficulties when dealing with tensor object inputs. It also differs from the previously published work in [29], where all of the projected features are used for recognition.

4) The development of a solution to the gait recognizer by representing gait sequences as tensor samples and extracting discriminative features from them. This is a more natural approach that differs from the previously published works (i.e., [3]-[7]), where either silhouettes or heuristic features derived from silhouettes are used as features.

The rest of this paper is organized as follows: Section II introduces basic multilinear algebra notations, concepts and the notion of multilinear projection for dimensionality reduction. In Section III, the problem of MPCA is formulated and an iterative solution is presented. Initialization procedures, termination criteria, convergence and subspace dimensionality are discussed in detail. The connection to PCA and 2DPCA is illustrated. The computational aspects of the proposed framework are also discussed in this section. The problem of tensor object recognition is discussed in Section IV. Section V lists experiments on both synthetic data sets and true application data. Synthetic data sets are used to verify the properties 
of the proposed methodology while gait data sets are used to demonstrate performance on a recognition problem of particular importance. Finally, Section VI summarizes the major findings of this work.

\section{MUlTilineAR PROJECTION OF TENSOR OBJECTS}

This section briefly reviews some basic multilinear concepts used in the MPCA framework development and introduces the multilinear projection of tensor objects for the purpose of dimensionality reduction.

\section{A. Notations and basic multilinear algebra}

Table VII in Appendix A-A lists the fundamental symbols defined in this paper. The notations followed are those decreed by convention in the multilinear algebra, pattern recognition and adaptive learning literature. Thus, in this work, vectors are denoted by lowercase boldface letters, e.g., x; matrices by uppercase boldface, e.g., $\mathbf{U}$; and tensors by calligraphic letters, e.g., $\mathcal{A}$. Their elements are denoted with indices in brackets. Indices are denoted by lowercase letters and span the range from 1 to the uppercase letter of the index, e.g., $n=1,2, \ldots, N$. To indicate part of a vector/matrix/tensor, ":" denotes the full range of the corresponding index and $n_{1}: n_{2}$ denotes indices ranging from $n_{1}$ to $n_{2}$. Throughout this paper, the discussion is restricted to real-valued vectors, matrices and tensors since the targeted applications, such as holistic gait recognition using binary silhouettes, involve real data only. The extension to the complex valued data sets is out of the scope of this work and it will be the focus of a forthcoming paper.

An $N^{t h}$-order tensor is denoted as: $\mathcal{A} \in \mathbb{R}^{I_{1} \times I_{2} \times \ldots \times I_{N}}$. It is addressed by $N$ indices $i_{n}, n=$ $1, \ldots, N$, and each $i_{n}$ addresses the $n$-mode of $\mathcal{A}$. The $n$-mode product of a tensor $\mathcal{A}$ by a matrix $\mathbf{U} \in \mathbb{R}^{J_{n} \times I_{n}}$, denoted by $\mathcal{A} \times_{n} \mathbf{U}$, is a tensor with entries: $\left(\mathcal{A} \times_{n} \mathbf{U}\right)\left(i_{1}, \ldots, i_{n-1}, j_{n}, i_{n+1}, \ldots, i_{N}\right)=$ $\sum_{i_{n}} \mathcal{A}\left(i_{1}, \ldots, i_{N}\right) \cdot \mathbf{U}\left(j_{n}, i_{n}\right)$. The scalar product of two tensors $\mathcal{A}, \mathcal{B} \in \mathbb{R}^{I_{1} \times I_{2} \times \ldots \times I_{N}}$ is defined as: $<\mathcal{A}, \mathcal{B}>=\sum_{i_{1}} \sum_{i_{2}} \ldots \sum_{i_{N}} \mathcal{A}\left(i_{1}, i_{2}, \ldots, i_{N}\right) \cdot \mathcal{B}\left(i_{1}, i_{2}, \ldots, i_{N}\right)$ and the Frobenius norm of $\mathcal{A}$ is defined as $\|\mathcal{A}\|_{F}=\sqrt{\langle\mathcal{A}, \mathcal{A}\rangle}$. The $i_{n}^{\text {th }}$ " $n$-mode slice" of $\mathcal{A}$ is an $(N-1)^{t h}$-order tensor obtained by fixing the $n$-mode index of $\mathcal{A}$ to be $i_{n}: \mathcal{A}\left(:, \ldots,:, i_{n},:, \ldots,:\right)$. The " $n$-mode vectors" of $\mathcal{A}$ are defined as the $I_{n}$-dimensional vectors obtained from $\mathcal{A}$ by varying the index $i_{n}$ while keeping all the other indices fixed. A rank-1 tensor $\mathcal{A}$ equals to the outer product of $N$ vectors: $\mathcal{A}=\mathbf{u}^{(1)} \circ \mathbf{u}^{(2)} \circ \ldots \circ \mathbf{u}^{(N)}$, which means that $\mathcal{A}\left(i_{1}, i_{2}, \ldots, i_{N}\right)=\mathbf{u}^{(1)}\left(i_{1}\right) \cdot \mathbf{u}^{(2)}\left(i_{2}\right) \cdot \ldots \cdot \mathbf{u}^{(N)}\left(i_{N}\right)$ for all values of indices. Unfolding $\mathcal{A}$ along the $n$-mode is denoted as $\mathbf{A}_{(n)} \in \mathbb{R}^{I_{n} \times\left(I_{1} \times \ldots \times I_{n-1} \times I_{n+1} \times \ldots \times I_{N}\right)}$. The column vectors of $\mathbf{A}_{(n)}$ are the $n$-mode vectors of $\mathcal{A}$. Figure 1 illustrates the 1-mode (column mode) unfolding of a third-order tensor.

Following standard multilinear algebra, any tensor $\mathcal{A}$ can be expressed as the product:

$$
\mathcal{A}=\mathcal{S} \times{ }_{1} \mathbf{U}^{(1)} \times{ }_{2} \mathbf{U}^{(2)} \times \ldots \times{ }_{N} \mathbf{U}^{(N)}
$$




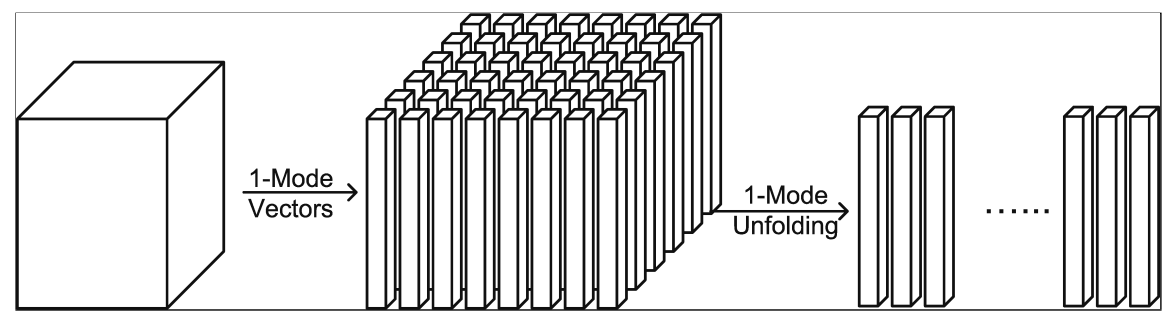

Fig. 1. Visual illustration of the 1-mode unfolding of a third-order tensor.

where $\mathcal{S}=\mathcal{A} \times{ }_{1} \mathbf{U}^{(1)^{T}} \times{ }_{2} \mathbf{U}^{(2)^{T}} \ldots \times_{N} \mathbf{U}^{(N)^{T}}$, and $\mathbf{U}^{(n)}=\left(\mathbf{u}_{1}^{(n)} \mathbf{u}_{2}^{(n)} \ldots \mathbf{u}_{I_{n}}^{(n)}\right)$ is an orthogonal $I_{n} \times I_{n}$ matrix. Since $\mathbf{U}^{(n)}$ has orthonormal columns, $\|\mathcal{A}\|_{F}^{2}=\|\mathcal{S}\|_{F}^{2}$ [1]. A matrix representation of this decomposition can be obtained by unfolding $\mathcal{A}$ and $\mathcal{S}$ as

$$
\mathbf{A}_{(n)}=\mathbf{U}^{(n)} \cdot \mathbf{S}_{(n)} \cdot\left(\mathbf{U}^{(n+1)} \otimes \mathbf{U}^{(n+2)} \otimes \ldots \otimes \mathbf{U}^{(N)} \otimes \mathbf{U}^{(1)} \otimes \mathbf{U}^{(2)} \otimes \ldots \otimes \mathbf{U}^{(n-1)}\right)^{T}
$$

where $\otimes$ denotes the Kronecker product. The decomposition can also be written as:

$$
\mathcal{A}=\sum_{i_{1}=1}^{I_{1}} \sum_{i_{2}=1}^{I_{2}} \ldots \sum_{i_{N}=1}^{I_{N}} \mathcal{S}\left(i_{1}, i_{2}, \ldots, i_{N}\right) \mathbf{u}_{i_{1}}^{(1)} \circ \mathbf{u}_{i_{2}}^{(2)} \circ \ldots \circ \mathbf{u}_{i_{N}}^{(N)},
$$

i.e., any tensor $\mathcal{A}$ can be written as a linear combination of $I_{1} \times I_{2} \times \ldots \times I_{N}$ rank-1 tensors. This decomposition is used in the following to formulate multilinear projection for dimensionality reduction.

\section{B. Tensor subspace projection for dimensionality reduction}

An $N^{t h}$-order tensor $\mathcal{X}$ resides in the tensor (multilinear) space $\mathbb{R}^{I_{1}} \otimes \mathbb{R}^{I_{2}} \ldots \otimes \mathbb{R}^{I_{N}}$, where $\mathbb{R}^{I_{1}}, \mathbb{R}^{I_{2}}$, $\ldots, \mathbb{R}^{I_{N}}$ are the $N$ vector (linear) spaces [23]. For typical image and video tensor objects such as 3D face images and gait sequences, although the corresponding tensor space is of high dimensionality, tensor objects typically are embedded in a lower dimensional tensor subspace (or manifold), in analogy to the (vectorized) face image embedding problem where vector image inputs reside in a low dimensional subspace of the original input space [33]. Thus, it is possible to find a tensor subspace that captures most of the variation in the input tensor objects and it can be used to extract features for recognition and classification applications. To achieve this objective, $P_{n}<I_{n}$ orthonormal basis vectors (principle axes) of the $n$-mode linear space $\mathbb{R}^{I_{n}}$ are sought for each mode $n$ and a tensor subspace $\mathbb{R}^{P_{1}} \otimes \mathbb{R}^{P_{2}} \ldots \otimes \mathbb{R}^{P_{N}}$ is formed by these linear subspaces. Let $\tilde{\mathbf{U}}^{(n)}$ denote the $I_{n} \times P_{n}$ matrix containing the $P_{n}$ orthornormal $n$-mode basis vectors. The projection of $\mathcal{X}$ onto the tensor subspace $\mathbb{R}^{P_{1}} \otimes \mathbb{R}^{P_{2}} \ldots \otimes \mathbb{R}^{P_{N}}$ is defined as $\mathcal{Y}=\mathcal{X} \times{ }_{1} \tilde{\mathbf{U}}^{(1)^{T}} \times{ }_{2} \tilde{\mathbf{U}}^{(2)^{T}} \ldots \times{ }_{N} \tilde{\mathbf{U}}^{(N)^{T}}$. 
The projection of an $n$-mode vector of $\mathcal{X}$ by $\tilde{\mathbf{U}}^{(n)^{T}}$ is computed as the inner product between the $n$ mode vector and the rows of $\tilde{\mathbf{U}}^{(n)^{T}}$. Figure 2 provides a visual illustration of the multilinear projection. In Fig. 2(a), a third-order tensor $\mathcal{A} \in \mathbb{R}^{10 \times 8 \times 6}$ is projected in the 1 -mode vector space by a projection matrix $\mathbf{B}^{(1)^{T}} \in \mathbb{R}^{5 \times 10}$, resulting in the projected tensor $\mathcal{A} \times{ }_{1} \mathbf{B}^{(1)^{T}} \in \mathbb{R}^{5 \times 8 \times 6}$. In the 1-mode projection, each 1-mode vector of $\mathcal{A}$ of length 10 is projected by $\mathbf{B}^{(1)^{T}}$ to obtain a vector of length 5 , as the differently shaded vectors indicate in Fig. 2(a). Similarly, Fig. 2(b) depicts the 2-mode and 3-mode vectors.

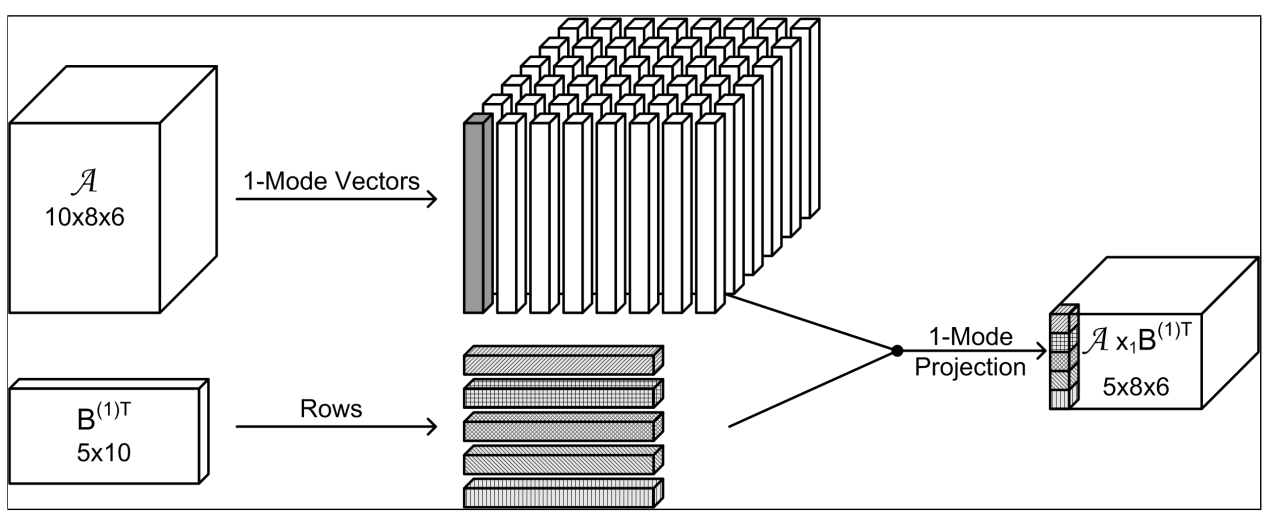

(a)

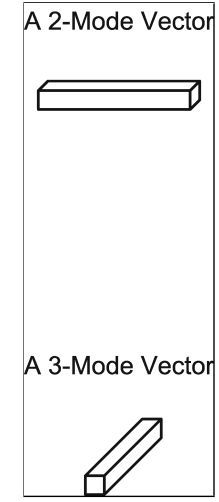

(b)

Fig. 2. Visual illustration of multilinear projection: (a) Projection in the 1-mode vector space. (b) 2-mode and 3-mode vectors.

\section{Multilinear Principal Component Analysis}

In this section, a multilinear principal component analysis (MPCA) solution to the problem of dimensionality reduction for tensor objects is introduced, researched and analyzed. Before formally stating the objective, the following definition is needed.

Definition 1: Let $\left\{\mathcal{A}_{m}, m=1, \ldots, M\right\}$ be a set of $M$ tensor samples in $\mathbb{R}^{I_{1}} \otimes \mathbb{R}^{I_{2}} \ldots \otimes \mathbb{R}^{I_{N}}$. The total scatter of these tensors is defined as: $\Psi_{\mathcal{A}}=\sum_{m=1}^{M}\left\|\mathcal{A}_{m}-\overline{\mathcal{A}}\right\|_{F}^{2}$, where $\overline{\mathcal{A}}$ is the mean tensor calculated as $\overline{\mathcal{A}}=\frac{1}{M} \sum_{m=1}^{M} \mathcal{A}_{m}$. The $n$-mode total scatter matrix of these samples is then defined as: $\mathbf{S}_{T_{\mathcal{A}}}^{(n)}=\sum_{m=1}^{M}\left(\mathbf{A}_{m(n)}-\overline{\mathbf{A}}_{(n)}\right)\left(\mathbf{A}_{m(n)}-\overline{\mathbf{A}}_{(n)}\right)^{T}$, where $\mathbf{A}_{m(n)}$ is the $n$-mode unfolded matrix of $\mathcal{A}_{m}$.

The statement above leads to the following formal definition of the problem to be solved:

A set of $M$ tensor objects $\left\{\mathcal{X}_{1}, \mathcal{X}_{2}, \ldots, \mathcal{X}_{M}\right\}$ is available for training. Each tensor object $\mathcal{X}_{m} \in$ $\mathbb{R}^{I_{1} \times I_{2} \times \ldots \times I_{N}}$ assumes values in a tensor space $\mathbb{R}^{I_{1}} \otimes \mathbb{R}^{I_{2}} \ldots \otimes \mathbb{R}^{I_{N}}$, where $I_{n}$ is the $n$-mode dimension of the tensor. The MPCA objective is to define a multilinear transformation $\left\{\tilde{\mathbf{U}}^{(n)} \in \mathbb{R}^{I_{n} \times P_{n}}, n=1, \ldots, N\right\}$ that maps the original tensor space $\mathbb{R}^{I_{1}} \otimes \mathbb{R}^{I_{2}} \ldots \otimes \mathbb{R}^{I_{N}}$ into a tensor subspace $\mathbb{R}^{P_{1}} \otimes \mathbb{R}^{P_{2}} \ldots \otimes \mathbb{R}^{P_{N}}$ (with $P_{n}<I_{n}$, for $\left.n=1, \ldots, N\right): \mathcal{Y}_{m}=\mathcal{X}_{m} \times{ }_{1} \tilde{\mathbf{U}}^{(1)^{T}} \times_{2} \tilde{\mathbf{U}}^{(2)^{T}} \ldots \times_{N} \tilde{\mathbf{U}}^{(N)^{T}}, m=1, \ldots, M$, such that 
$\left\{\mathcal{Y}_{m} \in \mathbb{R}^{P_{1}} \otimes \mathbb{R}^{P_{2}} \ldots \otimes \mathbb{R}^{P_{N}}, m=1, \ldots, M\right\}$ captures most of the variation observed in the original tensor objects, assuming that these variation are measured by the total tensor scatter.

In other words, the MPCA objective is the determination of the $N$ projection matrices $\left\{\tilde{\mathbf{U}}^{(n)} \in\right.$ $\left.\mathbb{R}^{I_{n} \times P_{n}}, n=1, \ldots, N\right\}$ that maximize the total tensor scatter $\Psi_{\mathcal{Y}}$ :

$$
\left\{\tilde{\mathbf{U}}^{(n)}, n=1, \ldots, N\right\}=\arg \max _{\tilde{\mathbf{U}}^{(1)}, \tilde{\mathbf{U}}^{(2)}, \ldots, \tilde{\mathbf{U}}^{(N)}} \Psi \mathcal{Y} \cdot
$$

Here, the dimensionality $P_{n}$ for each mode is assumed to be known or pre-determined. Discussions on the adaptive determination of $P_{n}$, when it is not known in advance, will be presented later.

\section{A. The MPCA algorithm}

To the best of the authors' knowledge, there is no known optimal solution which allows for the simultaneous optimization of the $N$ projection matrices. Since the projection to an $N^{t h}$-order tensor subspace consists of $N$ projections to $N$ vector subspaces, $N$ optimization subproblems can be solved by finding the $\tilde{\mathbf{U}}^{(n)}$ that maximizes the scatter in the $n$-mode vector subspace. This is discussed in the following theorem.

Theorem 1: Let $\left\{\tilde{\mathbf{U}}^{(n)}, n=1, \ldots, N\right\}$ be the solution to Equation (4). Then, for given all the other projection matrices $\tilde{\mathbf{U}}^{(1)}, \ldots, \tilde{\mathbf{U}}^{(n-1)}, \tilde{\mathbf{U}}^{(n+1)}, \ldots, \tilde{\mathbf{U}}^{(N)}$, the matrix $\tilde{\mathbf{U}}^{(n)}$ consists of the $P_{n}$ eigenvectors corresponding to the largest $P_{n}$ eigenvalues of the matrix

$$
\boldsymbol{\Phi}^{(n)}=\sum_{m=1}^{M}\left(\mathbf{X}_{m(n)}-\overline{\mathbf{X}}_{(n)}\right) \cdot \tilde{\mathbf{U}}_{\Phi^{(n)}} \cdot \tilde{\mathbf{U}}_{\Phi^{(n)}}^{T} \cdot\left(\mathbf{X}_{m(n)}-\overline{\mathbf{X}}_{(n)}\right)^{T}
$$

where

$$
\tilde{\mathbf{U}}_{\boldsymbol{\Phi}(n)}=\left(\tilde{\mathbf{U}}^{(n+1)} \otimes \tilde{\mathbf{U}}^{(n+2)} \otimes \ldots \otimes \tilde{\mathbf{U}}^{(N)} \otimes \tilde{\mathbf{U}}^{(1)} \otimes \tilde{\mathbf{U}}^{(2)} \otimes \ldots \tilde{\mathbf{U}}^{(n-1)}\right) .
$$

Proof: The proof of Theorem 1 is given in Appendix A-B.

Since the product $\tilde{\mathbf{U}}_{\Phi^{(n)}} \cdot \tilde{\mathbf{U}}_{\Phi^{(n)}}^{T}$ depends on $\tilde{\mathbf{U}}^{(1)}, \ldots, \tilde{\mathbf{U}}^{(n-1)}, \tilde{\mathbf{U}}^{(n+1)}, \ldots, \tilde{\mathbf{U}}^{(N)}$, the optimization of $\tilde{\mathbf{U}}^{(n)}$ depends on the projections in other modes and there is no closed form solution to this maximization problem. Instead, from Theorem 1, an iterative procedure can be utilized to solve (4), along the lines of the pseudo-code summarized in Fig. 3. The input tensors are centered first: $\left\{\tilde{\mathcal{X}}_{m}=\mathcal{X}_{m}-\overline{\mathcal{X}}, m=1, \ldots, M\right\}$. With initializations through full projection truncation (FPT), which is to be discussed in details in the following, the projection matrices are computed one by one with all the others fixed (local optimization). The local optimization procedure can be repeated, in a similar fashion as the alternating-least-squares (ALS) method [34], until the result converges or a maximum number $K$ of iterations is reached. 
Remark 1: The issue of centering has been ignored in the existing tensor processing literature. In the authors' opinion, the main reason for the apparent lack of studies on the problem of tensor data centering is due to the fact that previously published works focused predominately on tensor approximation and reconstruction. It should be pointed out that for the approximation/reconstruction problem, centering is not essential, as the (sample) mean is the main focus of attention. However, in recognition applications where the solutions involve eigenproblems, non-centering (in other words, an average different from zero) can potentially affect the per-mode eigen-decomposition and lead to a solution that captures the variation with respect to the origin rather than capturing the true variation of the data (with respect to the data center).

Remark 2: The effects of the ordering of the projection matrices to be computed have been studied empirically in this work and simulation results presented in Section $\mathrm{V}$ indicate that altering the ordering of the projection matrix computation does not result in significant performance differences in practical situations.

In the following subsections, several issues pertinent to the development and implementation of the MPCA algorithm are discussed. First, in-depth understanding of the MPCA framework is provided. The properties of full projection are analyzed, and the geometric interpretation of the $n$-mode eigenvalues is introduced together with the concept of eigentensor. In the sequence, the initialization method and the construction of termination criteria are described, and convergence issues are also discussed. Finally, methods for subspace dimensionality determination are proposed and the connection to PCA and 2DPCA is discussed, followed by computational issues.

\section{B. Full projection}

With respect to this analysis, the term full projection refers to the multilinear projection for MPCA with $P_{n}=I_{n}$ for $n=1, \ldots, N$. In this case, $\tilde{\mathbf{U}}_{\Phi^{(n)}} \cdot \tilde{\mathbf{U}}_{\boldsymbol{\Phi}^{(n)}}^{T}$ is an identity matrix, as it can be seen from the pertinent lemma listed in Appendix A-C. As a result, $\boldsymbol{\Phi}^{(n)}$ reduces to $\boldsymbol{\Phi}^{(n) *}=\sum_{m=1}^{M}\left(\mathbf{X}_{m(n)}-\overline{\mathbf{X}}_{(n)}\right)$. $\left(\mathbf{X}_{m(n)}-\overline{\mathbf{X}}_{(n)}\right)^{T}$, with $\boldsymbol{\Phi}^{(n) *}$ determined by the input tensor samples only and independent of other projection matrices. The optimal $\tilde{\mathbf{U}}^{(n)}=\mathbf{U}^{(n) *}$ is then obtained as the matrix comprised of the eigenvectors of $\boldsymbol{\Phi}^{(n) *}$ directly without iteration, and the total scatter $\Psi_{\mathcal{X}}$ in the original data are fully captured. However, there is no dimensionality reduction through this full projection. From the properties of eigendecomposition, it can be concluded that if all eigenvalues (per mode) are distinct, the full projection matrices (corresponding eigenvectors) are also distinct and that the full projection is unique (up to sign) [35]. 
Input: A set of tensor samples $\left\{\mathcal{X}_{m} \in \mathbb{R}^{I_{1} \times I_{2} \times \ldots \times I_{N}}, m=1, \ldots, M\right\}$.

Output: Low-dimensional representations $\left\{\mathcal{Y}_{m} \in \mathbb{R}^{P_{1} \times P_{2} \times \ldots \times P_{N}}, m=1, \ldots, M\right\}$ of the input tensor samples with maximum variation captured.

\section{Algorithm:}

Step 1 (Preprocessing): Center the input samples as $\left\{\tilde{\mathcal{X}}_{m}=\mathcal{X}_{m}-\overline{\mathcal{X}}, m=1, \ldots, M\right\}$, where $\overline{\mathcal{X}}=\frac{1}{M} \sum_{m=1}^{M} \mathcal{X}_{m}$ is the sample mean.

Step 2 (Initialization): Calculate the eigen-decomposition of $\boldsymbol{\Phi}^{(n) *}=\sum_{m=1}^{M} \tilde{\mathbf{X}}_{m(n)} \cdot \tilde{\mathbf{X}}_{m(n)}^{T}$ and set $\tilde{\mathbf{U}}^{(n)}$ to consist of the eigenvectors corresponding to the most significant $P_{n}$ eigenvalues, for $n=1, \ldots, N$.

Step 3 (Local optimization):

- Calculate $\left\{\tilde{\mathcal{Y}}_{m}=\tilde{\mathcal{X}}_{m} \times_{1} \tilde{\mathbf{U}}^{(1)^{T}} \times_{2} \tilde{\mathbf{U}}^{(2)^{T}} \ldots \times_{N} \tilde{\mathbf{U}}^{(N)^{T}}, m=1, \ldots, M\right\}$.

- Calculate $\Psi_{\mathcal{Y}_{0}}=\sum_{m=1}^{M}\left\|\tilde{\mathcal{Y}}_{m}\right\|_{F}^{2}$ (the mean $\overline{\tilde{Y}}$ is all zero since $\tilde{\mathcal{X}}_{m}$ is centered).

- For $k=1: K$

- For $n=1: N$

* Set the matrix $\tilde{\mathbf{U}}^{(n)}$ to consist of the $P_{n}$ eigenvectors of the matrix $\boldsymbol{\Phi}^{(n)}$, as defined in (5), corresponding to the largest $P_{n}$ eigenvalues.

- Calculate $\left\{\tilde{\mathcal{Y}}_{m}, m=1, \ldots, M\right\}$ and $\Psi_{\mathcal{Y}_{k}}$.

- If $\Psi_{\mathcal{Y}_{k}}-\Psi_{\mathcal{Y}_{k-1}}<\eta$, break and go to Step 4 .

Step 4 (Projection): The feature tensor after projection is obtained as $\left\{\mathcal{Y}_{m}=\mathcal{X}_{m} \times{ }_{1} \tilde{\mathbf{U}}^{(1)^{T}} \times{ }_{2} \tilde{\mathbf{U}}^{(2)^{T}} \ldots \times{ }_{N} \tilde{\mathbf{U}}^{(N)^{T}}, m=\right.$ $1, \ldots, M\}$.

Fig. 3. The pseudo-code implementation of the proposed MPCA algorithm.

To interpret the geometric meanings of the $n$-mode eigenvalues, the total scatter tensor $\mathcal{Y}_{\text {var }}^{*} \in$ $\mathbb{R}^{I_{1} \times I_{2} \times \ldots \times I_{N}}$ of the full projection is introduced as an extension of the total scatter matrix [36]. Each entry of the tensor $\mathcal{Y}_{v a r}^{*}$ is defined as below:

$$
\mathcal{Y}_{\text {var }}^{*}\left(i_{1}, i_{2}, \ldots, i_{N}\right)=\sum_{m=1}^{M}\left[\left(\mathcal{Y}_{m}^{*}-\overline{\mathcal{Y}}^{*}\right)\left(i_{1}, i_{2}, \ldots, i_{N}\right)\right]^{2},
$$

where $\mathcal{Y}_{m}^{*}=\mathcal{X}_{m} \times{ }_{1} \mathbf{U}^{(1) *^{T}} \ldots \times_{N} \mathbf{U}^{(N) *^{T}}$ and $\overline{\mathcal{Y}}^{*}=\frac{1}{M} \sum_{m=1}^{M} \mathcal{Y}_{m}^{*}$. Using the above definition, it can be shown that for the so-called full projection $\left(P_{n}=I_{n}\right.$ for all $\left.n\right)$, the $i_{n}^{t h} n$-mode eigenvalue $\lambda_{i_{n}}^{(n) *}$ is the sum of all the entries of the $i_{n}^{t h} n$-mode slice of $\mathcal{Y}_{v a r}^{*}$.

$$
\lambda_{i_{n}}^{(n) *}=\sum_{i_{1}=1}^{I_{1}} \ldots \sum_{i_{n-1}=1}^{I_{n-1}} \sum_{i_{n+1}=1}^{I_{n+1}} \ldots \sum_{i_{N}=1}^{I_{N}} \mathcal{Y}_{v a r}^{*}\left(i_{1}, \ldots, i_{n-1}, i_{n}, i_{n+1}, \ldots, i_{N}\right) .
$$

In this paper, the eigenvalues are all arranged in a decreasing order. Figure 4 shows visually what the $n$-mode eigenvalues represent. In this graph, third-order tensors, e.g. short sequences ( 3 frames) of images 
with size $5 \times 4$, are projected to a tensor space of size $5 \times 4 \times 3$ (full projection) so that a total scatter tensor $\mathcal{Y}_{\text {var }}^{*} \in \mathbb{R}^{5 \times 4 \times 3}$ is obtained.

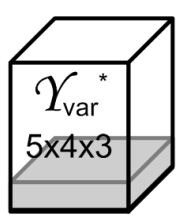

(a)

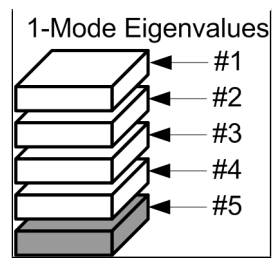

(b)

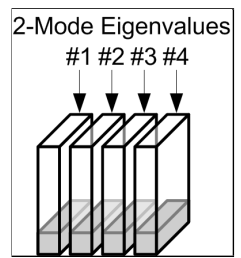

(c)

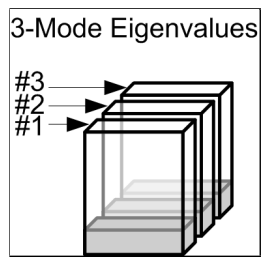

(d)

Fig. 4. Visual illustration of: (a) Total scatter tensor. (b) 1-mode eigenvalues. (c) 2-mode eigenvalues. (d) 3-mode eigenvalues.

Using equation (3), each tensor $\mathcal{X}_{m}$ can be written as a linear combination of $P_{1} \times P_{2} \times \ldots \times P_{N}$ rank-1 tensors $\tilde{\mathcal{U}}_{p_{1} p_{2} \ldots p_{N}}=\tilde{\mathbf{u}}_{p_{1}}^{(1)} \circ \tilde{\mathbf{u}}_{p_{2}}^{(2)} \circ \ldots \circ \tilde{\mathbf{u}}_{p_{N}}^{(N)}$. These rank-1 tensors will be called, hereafter, eigentensors. Thus, the projected tensor $\mathcal{Y}_{m}^{*}$ can be viewed as the projection onto these eigentensors, with each entry of $\mathcal{Y}_{m}^{*}$ corresponding to one eigentensor. These definitions and illustrations for MPCA help the understanding of the MPCA framework in the following discussions.

\section{Initialization by full projection truncation}

Full projection truncation (FPT) is used to initialize the iterative solution for MPCA, where the first $P_{n}$ columns of the full projection matrix $\mathbf{U}^{(n) *}$ is kept to give an initial projection matrix $\tilde{\mathbf{U}}^{(n)}$. The corresponding total scatter is denoted as $\Psi_{\mathcal{Y}_{0}}$ and this initialization is equivalent to the HOSVD-based solution in [28]. Although this FPT initialization is not the optimal solution to (4), it is bounded and is considered a good starting point for the iterative procedure, as will be discussed below.

Remark 3: There are other choices of initialization such as truncated identity matrices [20], [23], [31] (named as pseudo identity matrices) and random matrices. Simulation studies (reported in Section V) indicate that although in practical applications, the initialization step may not have a significant impact in terms of performance, it can affect the speed of convergence of the iterative solution. Since FPT results in much faster convergence, it is the one utilized throughout this work for initialization purposes.

In studying the optimality, with respect to (4), of the initialization procedure, let us assume, without loss of generality, that the 1-mode eigenvectors are truncated, in other words, only the first $P_{1}<I_{1}$ 1-mode eigenvectors are kept. In this case, the following theorem applies.

Theorem 2: Let $\mathbf{U}^{(n) *}$ and $\lambda_{i_{n}}^{(n) *}, i_{n}=1, \ldots, I_{n}$, be the matrix of the eigenvectors of $\boldsymbol{\Phi}^{(n) *}$ and the eigenvalues of $\boldsymbol{\Phi}^{(n) *}$, respectively, and $\tilde{\mathcal{Y}}_{m}=\tilde{\mathcal{X}}_{m} \times{ }_{1} \mathbf{U}^{(1) *^{T}} \ldots \times_{N} \mathbf{U}^{(N) *^{T}}, m=1, \ldots, M$. Keep only the 
first $P_{1}<I_{1}$ eigenvectors with $\sum_{i_{1}=P_{1}+1}^{I_{1}} \lambda_{i_{1}}^{(1) *}>0$ to get $\check{\mathcal{X}}_{m}=\check{\mathcal{Y}}_{m} \times_{1} \check{\mathbf{U}}^{(1)} \times_{2} \mathbf{U}^{(2) *} \ldots \times_{N} \mathbf{U}^{(N) *}$, where $\check{\mathcal{Y}}_{m}=\tilde{\mathcal{Y}}_{m}\left(1: P_{1},:, \ldots,:\right)$ and $\check{\mathbf{U}}^{(1)}=\mathbf{U}^{(1) *}\left(:, 1: P_{1}\right)$. Let $\check{\mathbf{\Phi}}^{(n)}$ correspond to $\check{\mathcal{X}}_{m}$, and the matrix of its eigenvectors and its eigenvalues be $\hat{\mathbf{U}}^{(n)}$ and $\hat{\lambda}_{i_{n}}^{(n)}$, respectively. Then,

$$
\hat{\lambda}_{i_{1}}^{(1)}= \begin{cases}\lambda_{i_{1}}^{(1) *}, & i_{1}=1, \ldots, P_{1} \\ 0, & i_{1}=P_{1}+1, \ldots, I_{1} .\end{cases}
$$

For $n>1$ (other modes), $\hat{\lambda}_{i_{n}}^{(n)} \leq \lambda_{i_{n}}^{(n) *}$. Furthermore, for each mode, at least for one value of $i_{n}$, $\hat{\lambda}_{i_{n}}^{(n)}<\lambda_{i_{n}}^{(n) *}$.

Proof: The proof is given in Appendix A-D.

It can be seen from Theorem 2 that if a non-zero eigenvalue is truncated in one mode, the eigenvalues in all the other modes tend to decrease in magnitude and the corresponding eigenvectors change accordingly. Thus, the eigen-decomposition needs to be recomputed in all the other modes, i.e., the projection matrices in all the other modes need to be updated. Since from Theorem 1, the computations of all the projection matrices are interdependent, the update of a projection matrix $\tilde{\mathbf{U}}^{\left(n^{*}\right)}$ updates the matrices $\left\{\tilde{\mathbf{U}}_{\boldsymbol{\Phi}^{(n)}}, n \neq n^{*}\right\}$ as well. Consequently, the projection matrices in all the other modes $\left\{\tilde{\mathbf{U}}^{(n)}, n \neq n^{*}\right\}$ are no longer consisting of the eigenvectors of the corresponding (updated) $\Phi^{(n)}$ and they need to be updated. The update continues until the termination criterion, which is discussed in the next subsection, is satisfied.

Figure 4 provides a visual illustration of Theorem 2. Removal of a basis vector in one mode results in eliminating a slice of $\mathcal{Y}_{v a r}^{*}$. In Fig. 4, if the last non-zero (fifth) 1-mode eigenvalue is discarded (shaded in Fig. 4(b)), the corresponding (fifth) 1-mode slice of $\mathcal{Y}_{v a r}^{*}$ is removed (shaded in Fig. 4(a)), resulting in a truncated total scatter tensor $\tilde{\mathcal{Y}}_{v a r}^{*} \in \mathbb{R}^{4 \times 4 \times 3}$. Discarding this slice will affect all eigenvalues in the remaining modes, whose corresponding slices have a non-empty overlap with the discarded 1-mode slice. In Figs. 4(c) and 4(d), the shaded part indicates the removed 1-mode slice corresponding to the discarded eigenvalue.

Having proven the non-optimality of FPT with respect to the objective function (4), we proceed to derive the bounds for FPT in the following theorem.

Theorem 3: Let $\lambda_{i_{n}}^{(n) *}$ denote the $i_{n}^{t h} n$-mode eigenvalue for the $n$-mode full projection matrix. The upper and lower bounds for $\left(\Psi_{\mathcal{X}}-\Psi_{\mathcal{Y}_{0}}\right)$, the loss of variation due to the FPT (measured by the total scatter), are derived as follows:

$$
\Psi_{L}=\max _{n} \sum_{i_{n}=P_{n}+1}^{I_{n}} \lambda_{i_{n}}^{(n) *} \leq\left(\Psi_{\mathcal{X}}-\Psi_{\mathcal{Y}_{0}}\right) \leq \sum_{n=1}^{N} \sum_{i_{n}=P_{n}+1}^{I_{n}} \lambda_{i_{n}}^{(n) *}=\Psi_{U} .
$$

Proof: The proof is given in Appendix A-E. 
From (9), it can be seen that the tightness of the bounds is determined by the eigenvalues in each mode. The bounds can be observed in Fig. 4. For instance, truncation of the last eigenvector in each of the three modes results in another truncated total scatter tensor $\hat{\mathcal{Y}}_{v a r}^{*} \in \mathbb{R}^{4 \times 3 \times 2}$, and thus the difference between $\Psi_{\mathcal{X}}$ and $\Psi_{\mathcal{Y}_{0}}$ (the sum of all entries in $\mathcal{Y}_{v a r}^{*}$ and $\hat{\mathcal{Y}}_{v a r}^{*}$, respectively) is upper-bounded by the total of the sums of all the entries in each truncated slice and lower-bounded by the maximum sum of all the entries in each truncated slice. For FPT, the gap between the actual loss of variation and the upper bound is due to the multiple counts of the overlaps between the discarded slice in one mode and the discarded slices in the other modes of $\mathcal{Y}_{v a r}^{*}$.

The tightness of the bounds $\Psi_{U}$ and $\Psi_{L}$ depends on the order $N$, the eigenvalue characteristics (distribution) such as the number of zero-valued eigenvalues, and the degree of truncation $P_{n}$. For example, for $N=1$, which is the case of PCA, $\Psi_{L}=\Psi_{U}$ and the FPT is the optimal solution so no iterations are necessary. Larger $N$ results in more terms in the upper bound and tends to lead to looser bound, and vice versa. In addition, if all the truncated eigenvectors correspond to zero-valued eigenvalues, $\Psi_{\mathcal{Y}_{0}}=\Psi_{\mathcal{X}}$ since $\Psi_{L}=\Psi_{U}=0$, and the FPT results in the optimal solution.

\section{Termination}

The termination criterion is to be determined in this work using the objective function $\Psi_{\mathcal{Y}}$. In particular, the iterative procedure terminates if $\left(\Psi_{\mathcal{Y}_{k}}-\Psi_{\mathcal{Y}_{k-1}}\right)<\eta$, where $\Psi_{\mathcal{Y}_{k}}$ and $\Psi_{\mathcal{Y}_{k-1}}$ are the resulted total scatter from the $k^{\text {th }}$ and $(k-1)^{\text {th }}$ iterations, respectively, and $\eta$ is a user-defined small number threshold (e.g., $\eta=10^{-6}$ ). In other words, the iterations stop if there is little improvement in the resulted total scatter (the objective function). In addition, the maximum number of iterations allowed is set to $K$ for computational consideration.

\section{E. Convergence of the MPCA algorithm}

The derivation of Theorem 1 (Appendix A-B) implies that per iteration, the total scatter $\Psi_{\mathcal{Y}}$ is a non-decreasing function (as it either remains the same or increases) since each update of the projection matrix $\tilde{\mathbf{U}}^{\left(n^{*}\right)}$ in a given mode $n^{*}$ maximizes $\Psi_{\mathcal{Y}}$, while the projection matrices in all the other modes, $\left\{\tilde{\mathbf{U}}^{(n)}, n \neq n^{*}\right\}$, are considered fixed. On the other hand, $\Psi_{\mathcal{Y}}$ is upper-bounded by $\Psi_{\mathcal{X}}$ (the variation in the original samples) since the projection matrices $\left\{\tilde{\mathbf{U}}^{(n)}\right\}$ consist of orthonormal columns. Therefore, MPCA is expected to have good convergence property. Empirical results presented in Section V indicate that the proposed MPCA algorithm converges very fast (within 5 iterations) for typical tensor objects. Furthermore, when per mode eigenvalues are all distinct (with multiplicity 1), which is the case for the 
simulated data as well as the gait data, the projection matrices $\left\{\tilde{\mathbf{U}}^{(n)}\right\}$, which maximize the objective function $\Psi_{\mathcal{Y}}$, is expected to converge as well. It should be noted that the claimed convergence regarding the projection matrices $\left\{\tilde{\mathbf{U}}^{(n)}\right\}$ is under the condition that the sign for the first component of each $n$ mode eigenvector is fixed since the eigenvector is unique up to sign. Simulation studies show that the projection matrices $\left\{\tilde{\mathbf{U}}^{(n)}\right\}$ do converge well.

\section{F. Determination of the tensor subspace dimensionality}

When the targeted dimensionality $\left\{P_{n}, n=1, \ldots, N\right\}$ is not specified in advance, its value has to be determined. Consequently, the objective function (4) needs to be revised to include a constraint on the desired dimensionality reduction. The revised objective function is as follows:

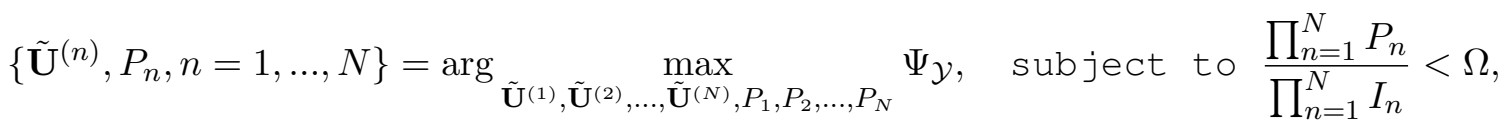

where the ratio between the targeted (reduced) dimensionality and the original tensor space dimensionality is utilized to measure the amount of dimensionality reduction, and $\Omega$ is a threshold to be specified by the user or determined based on empirical studies.

The proposed here tensor subspace dimensionality determination solution is called Sequential Mode Truncation (SMT). Starting with $P_{n}=I_{n}$ for all $n$ at $t=0$, at each subsequent step $t=t+1$, the SMT truncates, in a selected mode $n$, the $P_{n}^{\text {th }} n$-mode eigenvector of the reconstructed input tensors. The truncation could be interpreted as the elimination of the corresponding $P_{n}^{t h} n$-mode slice of the total scatter tensor. For the mode selection, the scatter loss rate $\delta_{t}^{(n)}$ due to the truncation of its $P_{n}^{t h}$ eigenvector is calculated for each mode. $\delta_{t}^{(n)}$ is defined as follows:

$$
\delta_{t}^{(n)}=\frac{\Psi_{\mathcal{Y}_{(t)}}-\Psi_{\mathcal{Y}_{(t-1)}}}{\left[P_{n} \cdot \prod_{j=1, j \neq n}^{N} P_{j}\right]-\left[\left(P_{n}-1\right) \cdot \prod_{j=1, j \neq n}^{N} P_{j}\right]}=\frac{\tilde{\lambda}_{P_{n}}^{(n)}}{\prod_{j=1, j \neq n}^{N} P_{j}},
$$

where $\Psi_{\mathcal{Y}_{(t)}}$ is the scatter obtained at step $t, \Pi_{j=1, j \neq n}^{N} P_{j}$ is the amount of dimensionality reduction achieved, and $\tilde{\lambda}_{P_{n}}^{(n)}$, which is the corresponding $P_{n}^{t h} n$-mode eigenvalue, is the loss of variation due to truncating the $P_{n}^{t h} n$-mode eigenvector. The mode with the smallest $\delta_{t}^{(n)}$ is selected for the step- $t$ truncation. For the selected mode $n, P_{n}$ is decreased by 1: $P_{n}=P_{n}-1$ and $\frac{\prod_{n=1}^{N} P_{n}}{\prod_{n=1}^{N} I_{n}}<\Omega$ is tested. The truncation stops when $\frac{\prod_{n=1}^{N} P_{n}}{\prod_{n=1}^{N} I_{n}}<\Omega$ is satisfied. Otherwise, the input tensors are reconstructed according to (1) using the current truncated projection matrices and they are used to recompute the $n$-mode eigenvalues and eigenvectors corresponding to full projection. Since eigenvalues in other modes are affected by the eigenvector truncation in a given mode (see Theorem 2), it is reasonable to assume that a procedure such 
as the SMT, which takes into account this effect, constitutes a reasonable good choice for determining $P_{n}$ in the sense of (10).

The $Q$-based method, a suboptimal, simplified dimensionality determination procedure that requires no recomputation, can be also used in practice. Let us define the ratio $Q^{(n)}=\frac{\sum_{i_{n}=1}^{P_{n}} \lambda_{i_{n}}^{(n) *}}{\sum_{i_{n}=1}^{I_{n}} \lambda_{i_{n}}^{(n) *}}$ to be the remained portion of the total scatter in the $n$-mode after the truncation of the $n$-mode eigenvectors beyond the $P_{n}^{t h}$, where $\lambda_{i_{n}}^{(n) *}$ is the $i_{n}^{t h}$ full-projection $n$-mode eigenvalue. In the proposed $Q$-based method, the first $P_{n}$ eigenvectors are kept in the $n$-mode (for each $n$ ) so that: $Q^{(1)}=Q^{(2)}=\ldots=Q^{(N)}=Q$ (the equality can hold approximately since it is unlikely to find $P_{n}$ that gives the exact equality in practice). It should be noted that $\sum_{i_{n}=1}^{I_{n}} \lambda_{i_{n}}^{(n) *}=\Psi_{\mathcal{X}}$ for all $n$ since from Theorem 1, the total scatter for the full projection was given as: $\Psi_{\mathcal{Y}}^{*}=\Psi_{\mathcal{X}}=\sum_{m=1}^{M}\left\|\mathbf{Y}_{m(n)}-\overline{\mathbf{Y}}_{(n)}\right\|_{F}^{2}=\sum_{i_{n}=1}^{I_{n}} \lambda_{i_{n}}^{(n) *}, n=1, \ldots, N$. This method can be viewed as an extension of the dimensionality selection strategy in traditional PCA to the multilinear case. The reason behind this choice is that loss of variation is (approximately) proportional to the sum of the corresponding eigenvalues of the discarded eigenvectors. By discarding the least significant eigenvectors in each mode, the variation loss can be contained and a tighter lower bound for $\Psi_{\mathcal{Y}_{0}}$ is obtained. The empirical study reported in the experimental section indicated that the $Q$-based method provides results similar to those obtained by SMT (as measured in terms of the total scatter captured) and thus it can be safely used instead of the more computationally expensive SMT alternative.

\section{G. MPCA Vs PCA and 2DPCA solutions}

It is not difficult to see that the MPCA framework generalizes not only the classical PCA solution but also a number of the so-called two-dimensional PCA (2DPCA) algorithms.

Indeed, for $N=1$, the input samples are vectors $\left\{\mathbf{x}_{m} \in \mathbb{R}^{I_{1}}\right\}$. There is only one mode and MPCA is reduced to PCA. For dimensionality reduction purposes, only one projection matrix $\mathbf{U}$ is needed in order to obtain $\mathbf{y}_{m}=\mathbf{x}_{m} \times_{1} \mathbf{U}=\mathbf{U}^{T} \mathbf{x}_{m}$. In this case, there is only one $\boldsymbol{\Phi}^{(n)}=\boldsymbol{\Phi}^{(1)}=\sum_{m=1}^{M}\left(\mathbf{x}_{m}-\overline{\mathbf{x}}\right)$. $\left(\mathbf{x}_{m}-\overline{\mathbf{x}}\right)^{T}$, which is the total scatter matrix of the input samples in PCA [36]. The projection matrix maximizing the total scatter (variation) in the projected space is determined from the eigenvectors of $\boldsymbol{\Phi}^{(1)}$. Thus, MPCA subsumes PCA.

In the so-called 2DPCA solutions, input samples are treated as matrices, in other words secondorder tensors. Two (left and right) projection matrices are sought to maximize the captured variation in the projected space. With $N=2$, the proposed MPCA algorithm (Fig. 3) is equivalent to the 2DPCA solution of [22], with the exception of the initialization procedure and termination criterion. Other 2DPCA algorithms such as those discussed in [21] can be viewed as variations of the method in [22] and thus 
they can be considered special cases of MPCA when second-order tensors are considered.

\section{H. Computational issues}

Apart from the actual performance of any proposed algorithm, its computational complexity, memory requirements and storage needs are relative measures of its practicality and usefulness as they determine the required computing power and processing (execution) time. To this end, we examine MPCA-related computational issues in a fashion similar to that introduced in [20].

Since this is an iterative solution, the computational complexity analysis is performed for one iteration. For simplicity, it is assumed that $I_{1}=I_{2}=\ldots=I_{N}=\left(\prod_{n=1}^{N} I_{n}\right)^{\frac{1}{n}}=I$. From a computational complexity point of view, the most demanding steps are the formation of the matrices $\boldsymbol{\Phi}^{(n)}$, the eigendecomposition of $\boldsymbol{\Phi}^{(n)}$, and the computation of the multilinear projection $\tilde{\mathcal{Y}}_{m}$. It should be noted that the use of multilinear multiplication and unfolding in order to compute $\boldsymbol{\Phi}^{(n)}$ is more efficient comparing to the use of Kronecker products. The computations needed to determine $\boldsymbol{\Phi}^{(n)}$, the $P_{n}$ eigenvectors of $\Phi^{(n)}$ and $\tilde{\mathcal{Y}}_{m}$ are in order of $O\left(M N \cdot I^{(N+1)}\right)$ (upper bounded), $O\left(I^{3}\right)$ and $O\left(N \cdot I^{(N+1)}\right)$, respectively. The total complexity is $O\left((N+1) \cdot M N \cdot I^{(N+1)}+N * I^{3}\right)$.

In MPCA, $\overline{\mathcal{X}}$ and $\boldsymbol{\Phi}^{(n)}$ can be computed incrementally by reading $\mathcal{X}_{m}$ or $\tilde{\mathcal{X}}_{m}$ sequentially without loss of information. Hence, memory requirements for the MPCA algorithm can be as low as $O\left(\prod_{n=1}^{N} I_{n}\right)$ as MPCA computes the solution without requiring all data samples in the memory. This is a major advantage that MPCA enjoys over the HOSVD-based solutions [26], [28], which requires the formation of an $(N+1)^{t h}$-order tensor when the input tensor samples are of $N^{t h}$-order. This is of considerable importance in applications with large data sets as the size of the input database may lead to increased complexity and high memory storage requirement ${ }^{1}$.

MPCA compresses each tensor sample of size $\prod_{n=1}^{N} I_{n}$ to $\prod_{n=1}^{N} P_{n}$, and it needs $N$ matrices $\tilde{\mathbf{U}}^{(n)} \in$ $\mathbb{R}^{I_{n} \times P_{n}}$ for compression and decompression. Thus, it requires $\left(M \cdot \prod_{n=1}^{N} P_{n}+\sum_{n=1}^{N}\left(I_{n} \times P_{n}\right)\right)$ scalars in the reduced space by MPCA and the compression ratio is defined as: $C R=\frac{M \cdot \prod_{n=1}^{N} I_{n}}{M \cdot \prod_{n=1}^{N} P_{n}+\sum_{n=1}^{N}\left(I_{n} \times P_{n}\right)}$. In studying the subspace dimensionality determination performance in the experiments, algorithms are compared under the same $C R$.

\footnotetext{
${ }^{1}$ As an iterative solution, MPCA has a higher I/O cost than a non-iterative solution. Nevertheless, since solving for the projection matrices using MPCA is only in the training phase of the targeted recognition tasks, it can be done offline and the additional I/O (and computational) cost due to iterations are not considered a disadvantage of the proposed MPCA solution.
} 


\section{MPCA-BASED TENSOR OBJECT RECOGNITION}

The projection matrices $\left\{\tilde{\mathbf{U}}^{(n)}, n=1, \ldots, N\right\}$, obtained by maximizing the total scatter $\Psi_{\mathcal{Y}}$ of the projected samples (in a reduced tensor space) from a set of training tensor samples $\left\{\mathcal{X}_{m}, m=1, \ldots, M\right\}$, can be used to extract features for various applications such as data compression, object retrieval, and pattern recognition. This section presents the MPCA-based tensor object recognition framework depicted in Fig. 5. In typical pattern recognition problems, such as human identification using fingerprints, face or gait signals, there are two types of data sets: the gallery and the probe [7]. The gallery set contains the set of data samples with known identities and it is used for training. The probe set is the testing set where data samples of unknown identity are to be identified and classified via matching with corresponding entries in the gallery set.

\section{A. Preprocessing}

MPCA accepts tensor samples of the same dimensions $I_{1} \times I_{2} \times \ldots \times I_{N}$ for feature extraction. However, in practice, tensor object samples are often of different dimensions. Therefore, the input tensors need to be normalized to standard dimensions first, and if they are noisy, a noise-removing preprocessing procedure could follow. The normalized tensor samples are then centered by subtracting the mean obtained from the gallery tensors.

\section{B. Feature extraction}

From the gallery set, a set of eigentensors is obtained, with reduced dimensionality $\left(P_{n} \leq I_{n}\right)$ determined by a user-specified $Q$, and each entry of a projected tensor feature can be viewed as a (scalar) feature corresponding to a particular eigentensor. Some of the small variation and noise are removed in the projection. For recognition, it should be noted that MPCA is an unsupervised technique without taking class labels into account. As a result, the variation captured in the projected tensor subspace includes both the within-class variation and the between-class variation. In the task of classification, a larger betweenclass variation relative to the within-class variation indicates good class separability, while a smaller between-class variation relative to the within-class variation indicates poor class separability. Hence, a feature selection strategy is proposed to select eigentensors according to their class discrimination power [30], [36], [37], which is defined to be the ratio of the between-class scatter over the within-class scatter:

Definition 2: The class discriminability $\Gamma_{p_{1} p_{2} \ldots p_{N}}$ for an eigentensor $\tilde{\mathcal{U}}_{p_{1} p_{2} \ldots p_{N}}$ is defined as

$$
\Gamma_{p_{1} p_{2} \ldots p_{N}}=\frac{\sum_{c=1}^{C} N_{c} \cdot\left[\overline{\mathcal{Y}}_{c}\left(p_{1}, p_{2}, \ldots, p_{N}\right)-\overline{\mathcal{Y}}\left(p_{1}, p_{2}, \ldots, p_{N}\right)\right]^{2}}{\sum_{m=1}^{M}\left[\mathcal{Y}_{m}\left(p_{1}, p_{2}, \ldots, p_{N}\right)-\overline{\mathcal{Y}}_{c_{m}}\left(p_{1}, p_{2}, \ldots, p_{N}\right)\right]^{2}},
$$


where $C$ is the number of classes, $M$ is the number of samples in the gallery set, $N_{c}$ is the number of samples for class $c$ and $c_{m}$ is the class label for the $m^{\text {th }}$ gallery sample $\mathcal{X}_{m}$. $\mathcal{Y}_{m}$ is the feature tensor of $\mathcal{X}_{m}$ in the projected tensor subspace. The mean feature tensor $\overline{\mathcal{Y}}=\frac{1}{M} \sum_{m} \mathcal{Y}_{m}$ and the class mean feature tensor $\overline{\mathcal{Y}}_{c}=\frac{1}{N_{c}} \sum_{m, c_{m}=c} \mathcal{Y}_{m}$.

For the eigentensor selection, the entries in the projected tensor $\mathcal{Y}_{m}$ (from the gallery set) are rearranged into a feature vector $\mathbf{y}_{m}$, ordered according to $\Gamma_{p_{1} p_{2} \ldots p_{N}}$ in descending order, and only the first $H_{\mathbf{y}}$ most discriminative components of $\mathbf{y}_{m}$ are kept for classification, with $H_{\mathbf{y}}$ determined empirically or userspecified. By this selection, a more discriminating subspace is resulted compared to the MPCA projected tensor subspace that includes both features with good separability and features with poor separability. Next, a weight tensor $\mathcal{W}$ is formed with entries defined as $\mathcal{W}\left(p_{1}, p_{2}, \ldots, p_{N}\right)=\sqrt{\Pi_{n=1}^{N} \lambda_{p_{n}}^{(n)}}$, where $\lambda_{p_{n}}^{(n)}$ denotes the $p_{n}^{t h} n$-mode eigenvalue corresponding to the projection matrix $\tilde{\mathbf{U}}^{(n)} . \mathcal{W}$ is rearranged into a vector $\mathbf{w}$ in the same order as $\mathbf{y}_{m}$ and only the first $H_{\mathbf{y}}$ components of $\mathbf{w}$ will be used in the next section as weights in measuring distances.

The feature vector $\mathbf{y}_{m}$ can be used directly for recognition, and a classical linear discriminant analysis (LDA) can also be applied to obtain an MPCA+LDA approach for recognition [36], similar to the popular approach of PCA+LDA. LDA seeks a projection $\mathbf{V}$ to maximize the ratio of the between-class scatter matrix $\mathbf{S}_{B}$ to the within-class scatter matrix $\mathbf{S}_{W}$, where $\mathbf{S}_{W}=\sum_{m=1}^{M}\left(\mathbf{y}_{m}-\overline{\mathbf{y}}_{c_{m}}\right)\left(\mathbf{y}_{m}-\overline{\mathbf{y}}_{c_{m}}\right)^{T}$ and $\overline{\mathbf{y}}_{c}=\frac{1}{N_{c}} \sum_{m, c_{m}=c} \mathbf{y}_{m}, \mathbf{S}_{B}=\sum_{c=1}^{C} N_{c}\left(\overline{\mathbf{y}}_{c}-\overline{\mathbf{y}}\right)\left(\overline{\mathbf{y}}_{c}-\overline{\mathbf{y}}\right)^{T}$, and $\overline{\mathbf{y}}=\frac{1}{M} \sum_{m} \mathbf{y}_{m}$. The solution $\mathbf{V}_{l d a}=$ $\arg \max _{\mathbf{V}} \frac{\left|\mathbf{V}^{T} \mathbf{S}_{B} \mathbf{V}\right|}{\left|\mathbf{V}^{T} \mathbf{S}_{W} \mathbf{V}\right|}=\left[\mathbf{v}_{1} \mathbf{v}_{2} \ldots \mathbf{v}_{H_{\mathbf{z}}}\right]$, where $\left\{\mathbf{v}_{h_{\mathbf{z}}}, h_{\mathbf{z}}=1, \ldots, H_{\mathbf{z}}\right\}$ is the set of generalized eigenvectors of $\mathbf{S}_{B}$ and $\mathbf{S}_{W}$ corresponding to the $H_{\mathbf{z}}(\leq C-1)$ largest generalized eigenvalues $\left\{\lambda_{h_{\mathbf{z}}}, h_{\mathbf{z}}=1, \ldots, H_{\mathbf{z}}\right\}$ [38]: $\mathbf{S}_{B} \mathbf{v}_{h_{\mathbf{z}}}=\lambda_{h_{\mathbf{z}}} \mathbf{S}_{W} \mathbf{v}_{h_{\mathbf{z}}}, h_{\mathbf{z}}=1, \ldots, H_{\mathbf{z}}$. Thus, the discriminant feature vector $\mathbf{z}_{m}$ is obtained as: $\mathbf{z}_{m}=\mathbf{V}_{l d a}^{T} \mathbf{y}_{m}$.

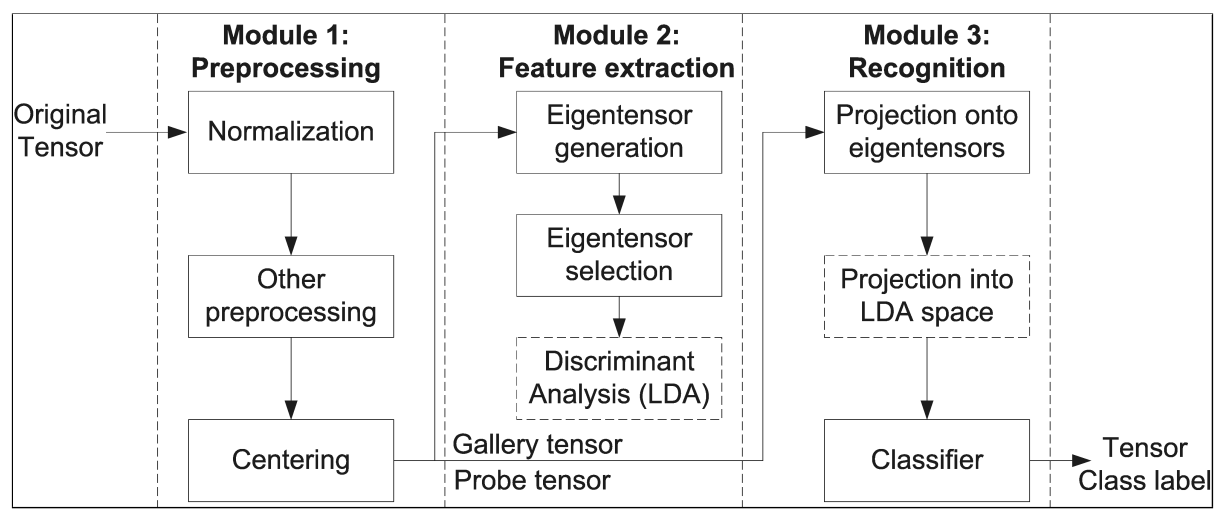

Fig. 5. Block diagram of the MPCA-based tensor object recognition system. 


\section{Feature classification}

In classification, the distance between feature vectors is of paramount importance as it determines the performance of the classification module. For the feature vectors discussed above $\left(\mathbf{y}_{m}\right.$ or $\left.\mathbf{z}_{m}\right)$, seven distance measures are adapted from [39] and tested: L1, L2, angle, modified Mahalanobis distance (MMD), modified L1 (ML1), modified L2 (ML2), and modified angle distance (MAD), as listed in Table I, where $\mathrm{g}$ is a weight vector. The first four measures are commonly used for measuring vector distances and the last three measures can be viewed as the weighted versions of the first three measures. If $\mathbf{z}_{m}$ is the feature vector, $H=H_{\mathbf{z}}$ and $\mathbf{g}(h)=\sqrt{\lambda_{h}}$, which is the simplified Mahalanobis distance introduced in [39]. If $\mathbf{y}_{m}$ is the feature vector, $H=H_{\mathbf{y}}$ and $\mathbf{g}=\mathbf{w}$, where $\mathbf{w}$ is defined above.

To measure the similarity of one test sample feature $\mathbf{y}$ (or $\mathbf{z}$ ) against $N_{c}$ sample features $\mathbf{y}_{n_{c}}$ (or $\mathbf{z}_{n_{c}}$ ) of a class $c$, the principle of nearest neighbor classifier is applied in this work. The matching score of $\mathbf{y}$ (or $\mathbf{z}$ ) with class $c$ is obtained as $S(\mathbf{y}, c)=-\min _{n_{c}} d\left(\mathbf{y}, \mathbf{y}_{n_{c}}\right)$ (or $S(\mathbf{z}, c)=-\min _{n_{c}} d\left(\mathbf{z}, \mathbf{z}_{n_{c}}\right)$ ), using one of the distance measures in Table I. Such a simple classifier is selected to study the performance mainly contributed by the MPCA-based feature extraction algorithm although better classifiers can be investigated.

TABLE I

SEVEN DistANCE MEASURES TESTED FOR MPCA-BASED TENSOR OBJECT RECOGNITION.

\begin{tabular}{|c|c|c|c|c|}
\hline Distance & L1 & L2 & Angle & MMD \\
\hline$d(\mathbf{a}, \mathbf{b})$ & $\sum_{h=1}^{H}|\mathbf{a}(h)-\mathbf{b}(h)|$ & $\sqrt{\sum_{h=1}^{H}[\mathbf{a}(h)-\mathbf{b}(h)]^{2}}$ & $-\frac{\sum_{h=1}^{H} \mathbf{a}(h) \cdot \mathbf{b}(h)}{\sqrt{\sum_{h=1}^{H} \mathbf{a}(h)^{2} \sum_{h=1}^{H} \mathbf{b}(h)^{2}}}$ & $-\sum_{h=1}^{H} \frac{\mathbf{a}(h) \cdot \mathbf{b}(h)}{\mathbf{g}(h)}$ \\
\hline \hline Distance & $\mathrm{ML} 1$ & $\mathrm{ML} 2$ & $\operatorname{MAD}$ & \\
\hline$d(\mathbf{a}, \mathbf{b})$ & $\sum_{h=1}^{H} \frac{|\mathbf{a}(h)-\mathbf{b}(h)|}{\mathbf{g}(h)}$ & $\sqrt{\sum_{h=1}^{H} \frac{[\mathbf{a}(h)-\mathbf{b}(h)]^{2}}{\mathbf{g}(h)}}$ & $-\frac{\sum_{h=1}^{H} \mathbf{a}(h) \cdot \mathbf{b}(h) / \mathbf{g}(h)}{\sqrt{\sum_{h=1}^{H} \mathbf{a}(h)^{2} \sum_{h=1}^{H} \mathbf{b}(h)^{2}}}$ & \\
\hline
\end{tabular}

Revision on July 30, 2010: A typo for the MAD was found in the published version and it is corrected here. We thank Dr. Irene Kotsia and Dr. Ioannis (Yiannis) Patras from the Queen Mary University of London for pointing this out.

\section{EXPERIMENTAL RESULTS}

This section summarizes a number of experiments performed here in support of the following objectives: 1) Investigate the various properties of the MPCA algorithm on synthetically generated as well as real application data sets; 2) Illustrate the efficacy of MPCA in tensor object classification, by applying MPCA to the very important problem of gait recognition and comparing its performance against state-of-the-art gait recognition algorithms as well as the baseline algorithm. 


\section{A. Experimentation using synthetic data}

Three synthetic data sets are constructed in order to study the behavior of the MPCA algorithm and in particular: a) the effects of the initial conditions, b) the effects of the ordering of the projection matrix computation, c) the evolution of the total scatter $\Psi_{\mathcal{Y}}$ over iterations, d) the number of iterations needed for convergence, and e) the performance of the tensor subspace dimensionality determination proposal.

1) Synthetic data generation: The core of the MPCA algorithm is the eigen-decomposition in each mode so the distribution of the eigenvalues is expected to impact significantly the performance of the algorithm. To study the MPCA properties on data of different characteristics, three synthetic data sets with eigenvalues in each mode spanning different magnitude ranges are generated. In particular, $M$ third order tensor samples $\mathcal{A}_{m} \in \mathbb{R}^{I_{1} \times I_{2} \times I_{3}}$ are generated per set according to $\mathcal{A}_{m}=\mathcal{B}_{m} \times{ }_{1} \mathbf{C}^{(1)} \times{ }_{2} \mathbf{C}^{(2)} \times{ }_{3} \mathbf{C}^{(3)}+\mathcal{D}_{m}$, using a core tensor $\mathcal{B}_{m} \in \mathbb{R}^{I_{1} \times I_{2} \times I_{3}}, n$-mode projection matrix $\mathbf{C}^{(n)} \in \mathbb{R}^{I_{n} \times I_{n}}(n=1,2,3)$ and a "noise" tensor $\mathcal{D}_{m} \in \mathbb{R}^{I_{1} \times I_{2} \times I_{3}}$. All entries in $\mathcal{B}_{m}$ are drawn from a zero-mean unit-variance Gaussian distribution

and are multiplied by $\left(\frac{I_{1} \cdot I_{2} \cdot I_{3}}{i_{1} \cdot i_{2} \cdot i_{3}}\right)^{f}$. In this data generation procedure, $f$ controls the eigenvalue distributions, so that data sets are created having eigenvalues' magnitudes in different ranges. Smaller $f$ results in a narrower range of eigenvalue spread. The matrices $\mathbf{C}^{(n)}(n=1,2,3)$ are orthogonal matrices obtained by applying singular value decomposition (SVD) on random matrices with entries drawn from zero-mean, unit-variance Gaussian distribution. All entries of $\mathcal{D}_{m}$ are drawn from a zero-mean Gaussian distribution with variance 0.01 . Three synthetic data sets, $\mathrm{db} 1, \mathrm{db} 2$ and $\mathrm{db} 3$, of size $30 \times 20 \times 10$ with $M=100$ and $f=1 / 2,1 / 4$, and $1 / 16$, respectively, are created. Figure 6 (a) depicts the spread of eigenvalue magnitudes and Fig. 6(b) depicts their eigenvalue cumulative distribution.

2) MPCA properties: First, the effects of the initial conditions are tested using the synthetic data sets. Both random matrices and pseudo identity matrices (truncated identity matrices) have been utilized. Typical examples are shown in Fig. 7. From the simulation studies, it can be observed that despite the different initializations, the algorithm, when applied on sets $\mathrm{db} 1$ and $\mathrm{db} 2$, converges to the same point within three iterations. On set $\mathrm{db} 3$, the algorithm with different initializations converges to the same point within ten iterations for $Q \geq 0.35$. For small value of $Q(<0.35)$ on set $\mathrm{db} 3$, the algorithm using random matrices as initialization could converge to a point that is different from (lower than) the point to which the algorithm using the other two initialization methods converges, as shown in Fig. 7(c), indicating that initialization methods could affect the final results on data sets with similar characteristics as db3 when a small $Q$ is used. In summary, initialization has little effect on the final results for synthetic data sets $\mathrm{db} 1$ and $\mathrm{db} 2$ with all values of $Q$, and for synthetic data set $\mathrm{db} 3$ with $Q \geq 0.35$. In pattern recognition 


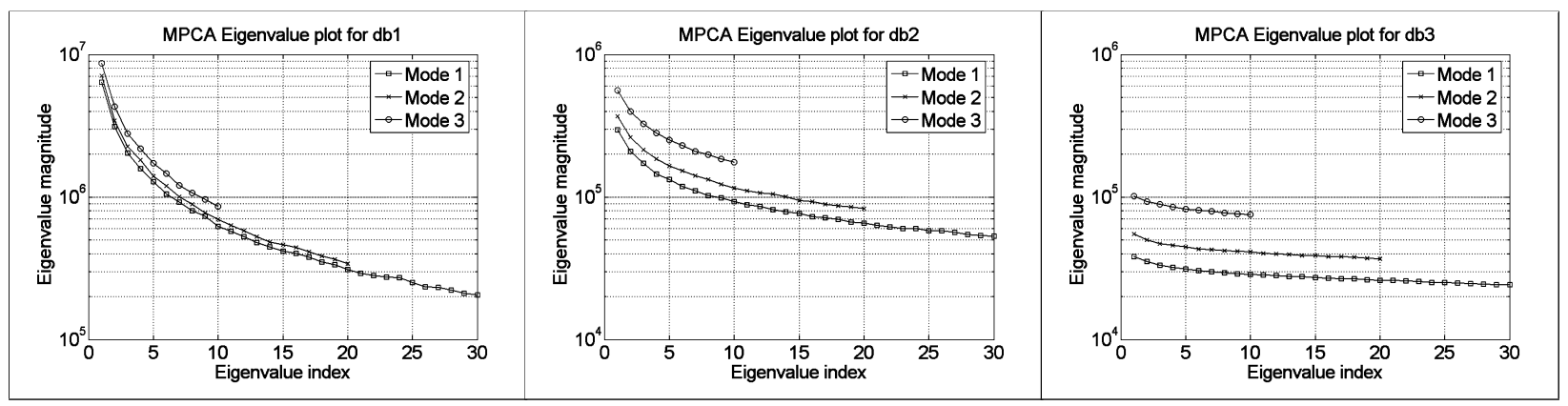

(a) The distribution of eigenvalues.

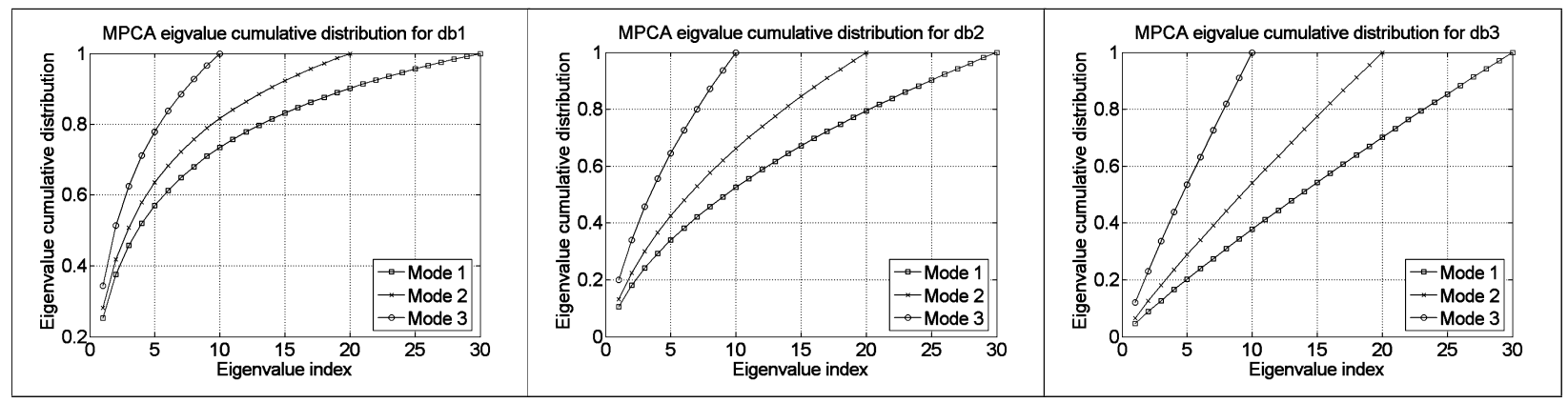

(b) The cumulative distribution of eigenvalues.

Fig. 6. The eigenvalue magnitudes and their cumulative distributions for the synthetic data sets: db1, db2 and db3.

applications, it is often desired to keep most of the variation/energy in the original data and hence the proposed algorithm using different initializations is expected to converge well since $Q>0.35$ is easily satisfied in practice. Since the algorithm using the proposed initialization, Full Projection Truncation (FPT), converges faster than the algorithm using the other initialization methods, the FPT is expected to be closer to the local maximum point and it is used for initialization in this work.

Second, the effects of the ordering of the projection matrix computation are studied through simulations on the synthetic data sets. Simulation results indicate that there is no significant difference in the captured total scatter for $\mathrm{db} 1$ and $\mathrm{db} 2$, while there is some small difference for $\mathrm{db} 3$. For $\mathrm{db} 3$, the difference in total scatter captured using different orderings is negligible $(<0.01 \%)$ for $Q>0.5$ and it increases as $Q$ decreases, e.g., the difference is about $1 \%$ when $Q=0.2$. This observation is consistent with the poorer convergence property of $\mathrm{db} 3$, especially for a small $Q$, in other experiments such as the initialization study above and the convergence study in the following.

To study the evolution of the total scatter $\Psi_{\mathcal{Y}}$ over iterations, the ratio of the value of $\Psi_{\mathcal{Y}_{k}}$ over the initial value $\Psi_{\mathcal{Y}_{0}}$ is plotted against the number of iterations, as a function of dimensionality reduction 


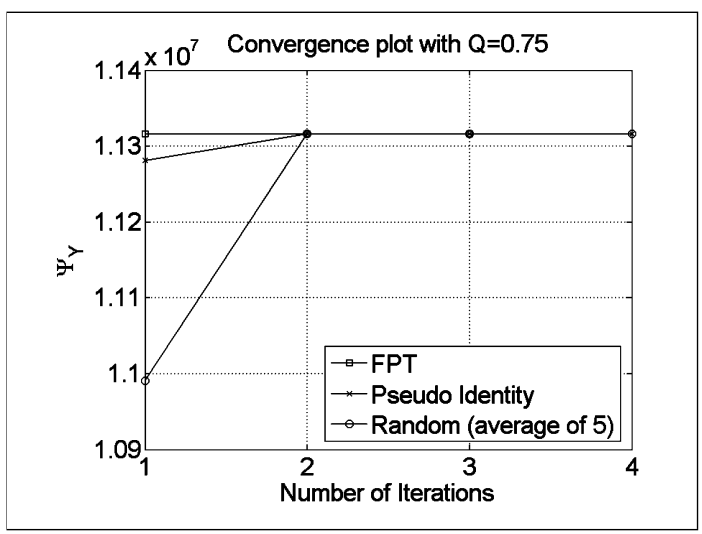

(a) Convergence plot for db1 with $Q=0.75$.

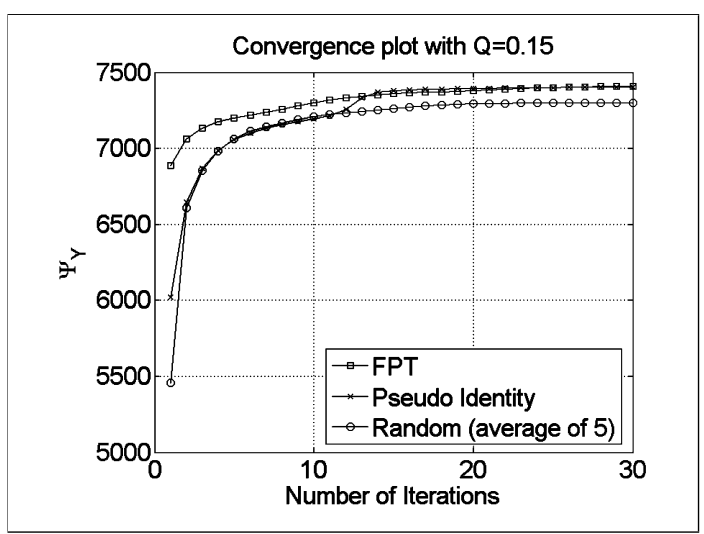

(c) Convergence plot for db3 with $Q=0.15$.

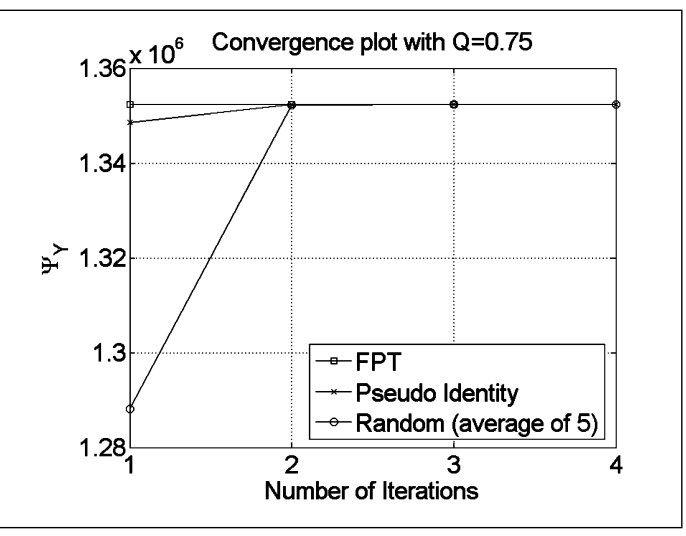

(b) Convergence plot for db2 with $Q=0.75$.

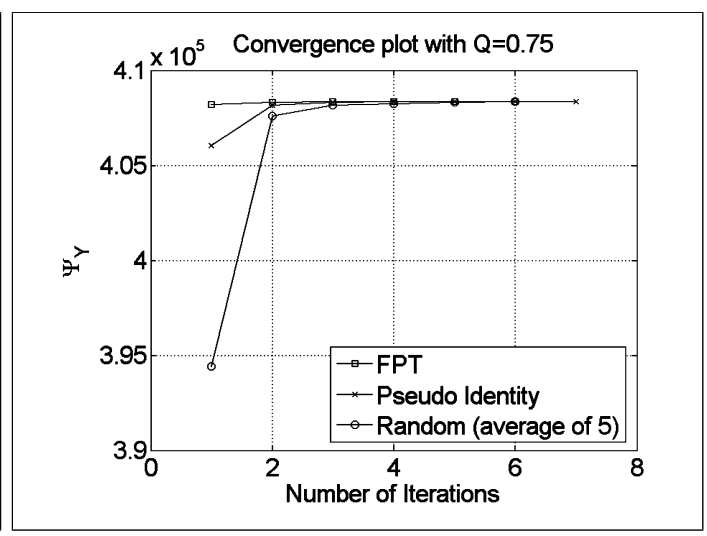

(d) Convergence plot for db3 with $Q=0.75$.

Fig. 7. Convergence plot for different initializations.

determined by $Q$. For illustration purpose, results obtained for up to 15 iterations with $Q=0.2$ and $Q=0.8$ are shown in Figs. 8(a) and 8(b), respectively. As it can be seen from the figure, the first iteration results in the greatest increase in $\Psi_{\mathcal{Y}}$ while subsequent iterations result in smaller and smaller increments, especially for data sets $\mathrm{db} 1$ and $\mathrm{db} 2$. To study the empirical convergence performance, the number of iterations for convergence using a termination value of $\eta=10^{-6}$ is plotted in Fig. 8(c) as a function of the parameter $Q$. These figures demonstrate that in MPCA, the number of iterations needed to converge decreases as the range spanned by the eigenvalues for the data samples or the value of $Q$ increases.

The dependency on $Q$ can be explained from two aspects. $Q$ is closely related to the number of eigenvectors truncated. First, from Theorem 3, the bounds on $\Psi_{\mathcal{Y}_{0}}$ tend to become looser when the number of eigenvectors truncated increases ( $Q$ decreases), and vice versa. Looser (tighter) bounds tend 
to result in a poorer (better) initialization and it takes more (less) iterations to reach a local optimum. Second, by Theorem 2, more truncation (smaller value of $Q$ ) tends to decrease the eigenvalues in the other modes more so that more iterations are needed to converge, and vice versa. The dependency on the range of eigenvalue spread is less obvious. Narrower range in a mode means the variation along each eigenvector in this mode is similar and each of the truncated eigenvectors tends to encode similar amount of variation as each remaining one, which tends to make the convergence harder. On the other hand, broader range of the eigenvalue spread in a mode means that the energy is more concentrated in this mode and in this case, convergence is expected to be easier.

In practical recognition tasks, $Q$ is commonly set to a large value in order to capture most of the variation. Furthermore, the eigenvalues of practical data samples usually spread a wide range in each mode due to redundancy/correlation. Therefore, in practice, the number of iterations can be set to a small value such as 1 with little sacrifice in the variation captured while enjoying significant gain in empirical efficiency. In the following experiments, there is only one iteration $(K=1)$ in MPCA and in this case, the calculation of $\Psi_{\mathcal{Y}_{k}}$ and $\tilde{\mathcal{Y}}_{m}$ is not needed in the local optimization (step 3) of Fig. 3.

To investigate the $Q$-based method and the SMT method for subspace dimensionality determination, $P_{n}$ is determined for each mode $n$ using these two methods with various degrees of truncation across all the modes. The resulted total scatters are compared under the same compression ratio $C R$ (the same reduced dimensionality), as shown in Fig. 8(d). The figure indicates that these two methods have very similar results for all the three data sets. Hence, in practice, the $Q$-based method, which is more efficient, is used to determine the tensor subspace dimensionality. In addition, it is also verified through experiments, which are not included here due to space limitation, that truncation in all modes is advantageous against truncation in only one or two modes.

\section{B. MPCA-based gait recognition}

The emerging application of video-based gait recognition is of particular interest in the context of surveillance-at-a-distance applications [3]. Gait video is naturally a third-order tensor with the column, row and time mode. Most of the gait recognition algorithms proposed in the literature extract features using frame-by-frame processing and they did not fully utilize the natural spatial and temporal correlations of the input data. Unfortunately, the linear subspace algorithms that have been successfully applied, adapted or developed in other biometrics applications, such as faces [16], are not directly applicable to the gait recognition problem because the vectorization of gait video input results in vectors with much higher dimensions compared to that of vectorized face images, which are impossible to process. For example, 


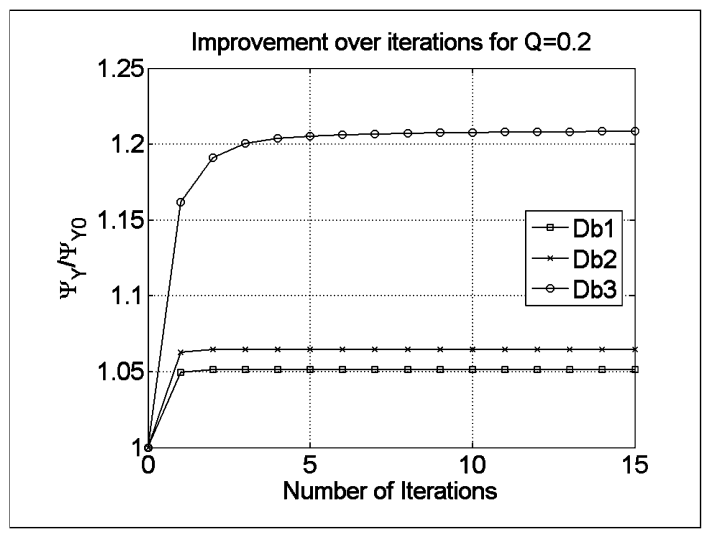

(a) Evolution of $\Psi_{\mathcal{Y}}$ for $Q=0.2$.

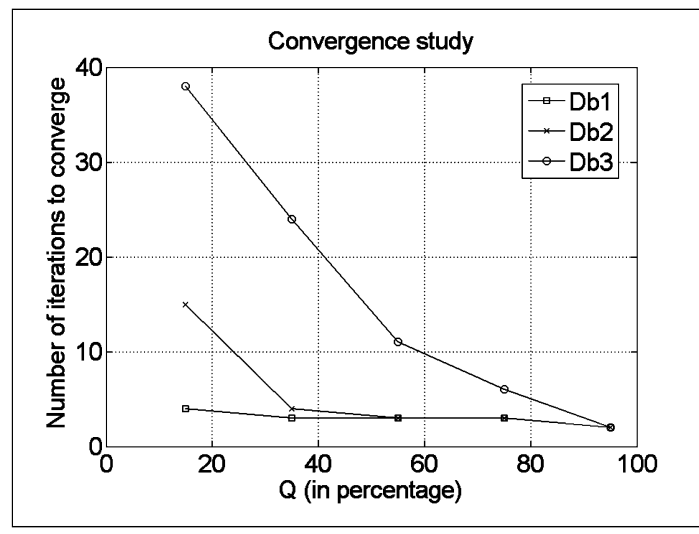

(c) Number of iterations to converge.

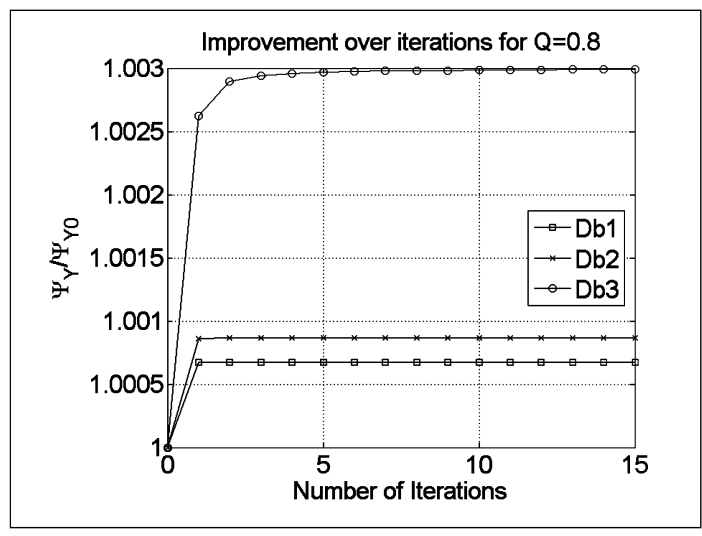

(b) Evolution of $\Psi_{\mathcal{Y}}$ for $Q=0.8$.

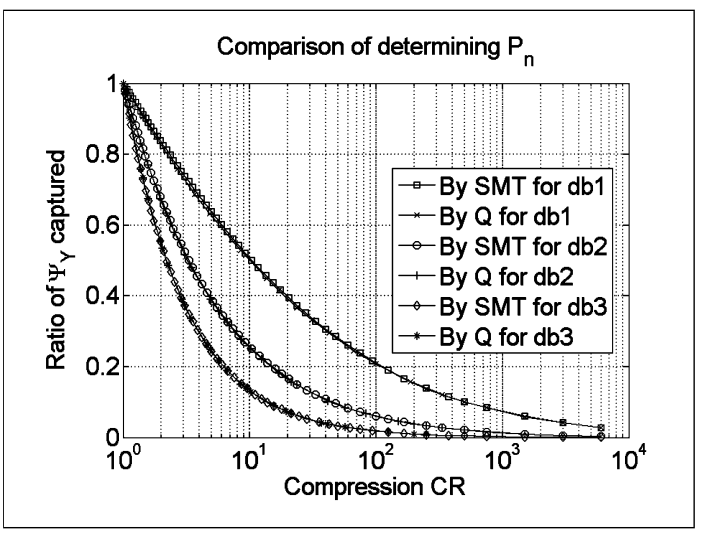

(d) SMT Vs. $Q$-based selection of $P_{n}$

Fig. 8. Illustration of various properties of MPCA on the synthetic data sets.

each gait silhouette sequence from the standard USF data sets has a frame size of $128 \times 88$ and each sequence contains approximately 180 frames. The dimensionality of such a sequence is approximately two orders of magnitude higher to that of the typical image dimensionality encountered in the NIST FERET face database [40]. By treating a number of frames as a single tensor object from which features are to be extracted, both the spatial and the temporal correlation of the original information is fully preserved, and thus a cost-effective feature extraction procedure can be obtained under the framework of the MPCA-based tensor object recognition in Sec. IV. With each gait half-cycle treated as a tensor sample, the proposed gait recognition solution introduces a novel representation called EigenTensorGait and it can be extended readily to other recognition problems as well. A gait recognition algorithm typically consists of the following steps: algorithm to partition a gait sequence into cycles (or half cycles), feature representation and extraction method, and the matching algorithm. In addition, preprocessing may be 
applied to further improve the quality of silhouette images for better recognition results, which is not included in this work.

1) The Gait Challenge data sets: The experiments are carried out on the USF HumanID "Gait Challenge" data sets version 1.7 [7]. This database consists of 452 sequences from 74 subjects (persons) walking in elliptical paths in front of the camera. For each subject, there are three covariates: viewpoint (left/right), shoe type (two different types) and surface type (grass/concrete). The gallery set contains 71 sequences (subjects) and seven experiments (probe sets) are designed for human identification as shown in Table II. The capturing condition for each probe set is summarized in brackets after the probe name in the Table, where C, G, A, B, L, and R stand for cement surface, grass surface, shoe type A, shoe type $\mathrm{B}$, left view, and right view, respectively. The capturing condition of the gallery set is GAR. Subjects are unique in the gallery and each probe set and there are no common sequences between the gallery set and any of the probe sets. Also, all the probe sets are distinct. A baseline algorithm is proposed in [7] to extract human silhouettes and recognize individuals in this database. The extracted silhouettes data provided by them is widely used in the literature. For this reason, it is considered as inputs to the experiments reported here in order to facilitate a fair comparison amongst the gait recognition algorithms considered here.

TABLE II

SEVEN EXPERIMENTS DESIGNED FOR HUMAN IDENTIFICATION IN USF HUMANID DATA SETS.

\begin{tabular}{|c|c|c|c|c|c|c|c|}
\hline Probe set & $\mathrm{A}(\mathrm{GAL})$ & $\mathrm{B}(\mathrm{GBR})$ & $\mathrm{C}(\mathrm{GBL})$ & $\mathrm{D}(\mathrm{CAR})$ & $\mathrm{E}(\mathrm{CBR})$ & $\mathrm{F}(\mathrm{CAL})$ & $\mathrm{G}(\mathrm{CBL})$ \\
\hline \hline Size & 71 & 41 & 41 & 70 & 44 & 70 & 44 \\
\hline \hline Difference & View & Shoe & Shoe, view & Surface & Surface, shoe & Surface, view & Surface, shoe, view \\
\hline
\end{tabular}

2) The EigenTensorGait representation: While in many recognition problems, an input sample is unambiguously defined, such as iris, face or fingerprint images, there is no obvious definition of a gait sample. The proposed method treats each half gait cycle as a data sample. Thus, the input is a 3rd-order tensor, and the spatial column space, row space and the time space account for its three modes, as shown in Fig. 9.

To obtain half cycles, a gait silhouette sequence is partitioned in a way similar to that used in [7]. The number of foreground pixels is counted in the bottom half of each silhouette since legs are the major visible moving body parts from a distance. This number will reach a maximum when the two legs are farthest apart and drop to a minimum when the legs overlap. The sequence of numbers is smoothed with a 


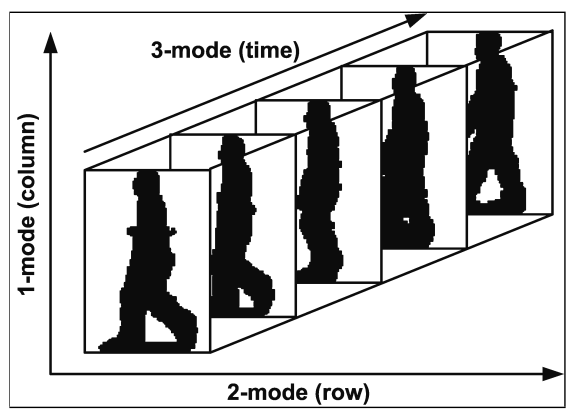

Fig. 9. Illustration of the gait silhouette sequence as a third-order tensor.

running average filter and the minimums in this number sequence partition the sequence into several half gait cycles. Following the definition above, there are 731 gait samples in the Gallery set and each subject has an average of roughly 10 samples available. This simple partition method may be improved further by taking the periodic nature of the gait cycles into account, such as the more robust cycle partitioning algorithm in [6], which is not investigated in this paper.

Each frame of the gait silhouette sequences from the USF data sets is of standard size $128 \times 88$, but the number of frames in each gait sample obtained through half cycle partition has some variation. Before feeding the gait samples to MPCA, the tensorial inputs need to be normalized to the same dimension in each mode. Since the row and column dimensions are normalized by default, only the time mode, i.e., the number of frames in each gait sample, is subject to normalization. The normalized time mode dimension is chosen to be 20 , which is roughly the average number of frames in each gait sample. Thus, each gait sample has a canonical representation of $I_{1} \times I_{2} \times I_{3}=128 \times 88 \times 20$, and feature vectors are extracted according to the method described in Sec.IV for the gait samples $\left\{\mathcal{X}_{m}, m=1, \ldots, M\right\}$. In the following, a simple procedure for this time-mode normalization is described.

Consider one sample $\mathcal{A} \in \mathbb{R}^{I_{1} \times I_{2} \times D_{3}}$. While there are sophisticated algorithms available, such as mapping a gait cycle to a unit circle using nonlinear interpolation [26], conventional interpolation algorithms, such as linear interpolation, can be applied to the time-mode normalization as well. In this work, each 3-mode (time-mode) vector is interpolated linearly to the normal size $I_{3} \times 1$, followed by binarization to get binary silhouettes.

The 1-mode unfolding, i.e., concatenating the frames of a sequence sequentially in a row, of a gait sample and the mean gait sample, are shown in Figs. 10(a) and 10(b), respectively. Figure 11 shows a plot of the eigenvalues in three modes and their cumulative distributions. The 2-mode eigenvalues drop sharply after the $20^{\text {th }}$ eigenvalue, indicating high redundancy in the 2-mode (row mode). The 1-mode 


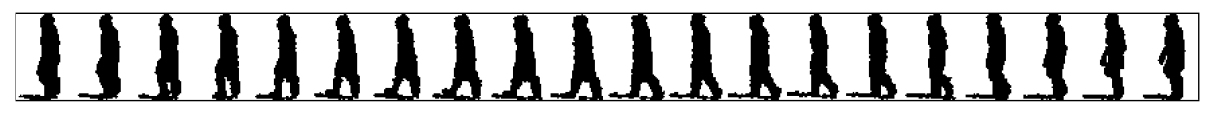

(a) 1-mode unfolding of a gait silhouette sample.

\section{IIINARARARALIIII}

(b) 1-mode unfolding of the mean of the gait silhouette samples.

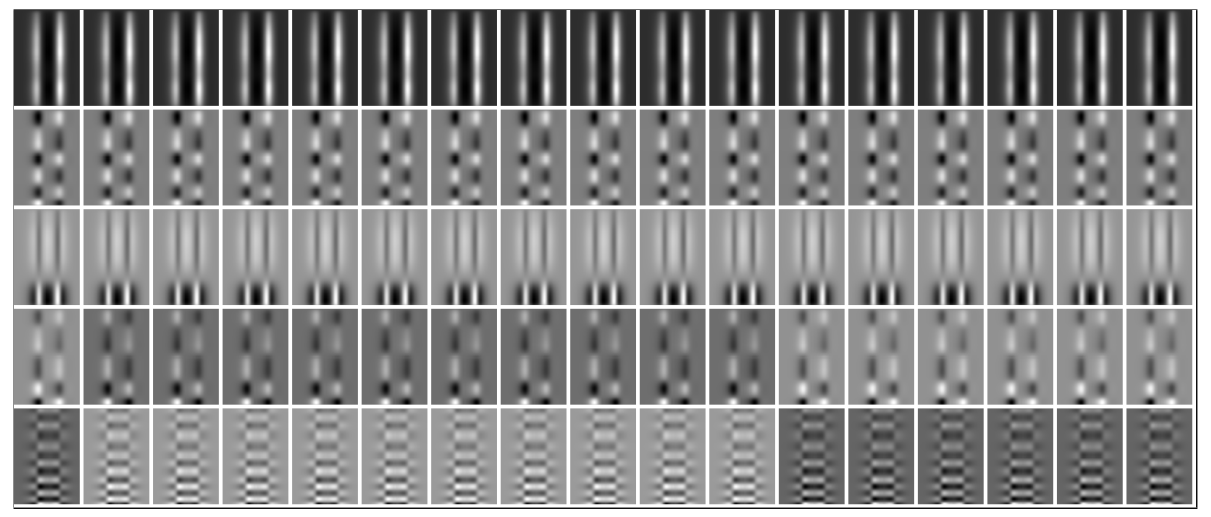

(c) The $1^{\text {st }}, 2^{\text {nd }}, 3^{r d}, 45^{\text {th }}$ and $92^{\text {th }}$ discriminative EigenTensorGaits.

Fig. 10. A gait sample, the mean gait sample, and a number of representative EigenTensorGaits.

(column mode) eigenvalues decrease gradually except at the last point, which may be due to the centering.
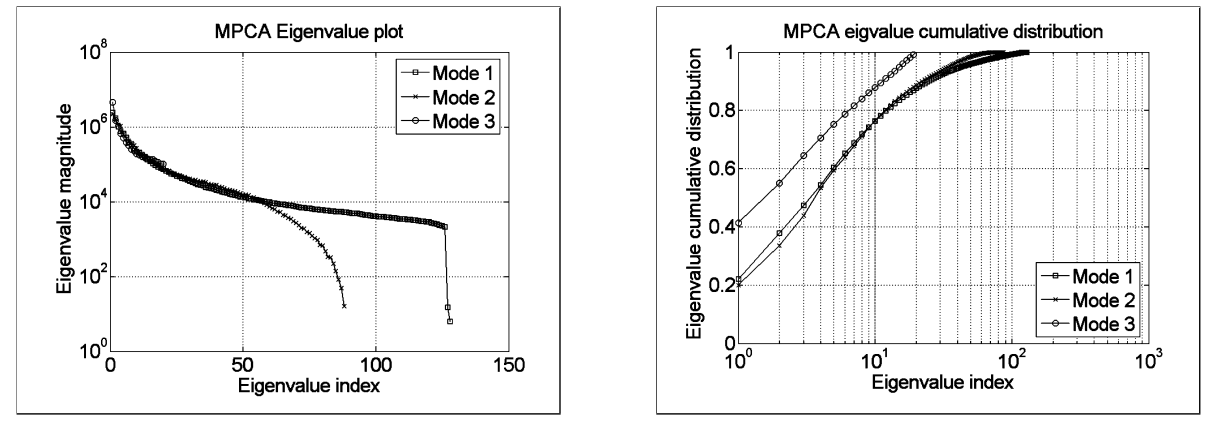

Fig. 11. The eigenvalue magnitudes and their cumulative distributions for the gallery set.

The eigentensors obtained from the gallery gait sequences through MPCA are named EigenTensorGaits. This approach to gait analysis is called hereafter the EigenTensorGait (ETG) approach and if LDA is subsequently applied, the approach is called the ETG+LDA (ETGLDA) approach. Figure 10(c) shows the 1-mode unfolding of the $1^{\text {st }}, 2^{\text {nd }}, 3^{r d}, 45^{\text {th }}$ and $92^{\text {th }}$ most discriminative EigenTensorGaits for illustration. From the figure, it is observed that the EigenTensorGaits act as a set of multiresolution filters, where 
each EigenTensorGait can be viewed as a filter, and the projection using them is very similar to a filtering process applying a filter bank in multiresolution analysis. Using the proposed EigenTensorGait representation, gait features are extracted from gait sequences for recognition.

3) Gait feature matching for recognition: In the ETG approach, to obtain the matching score of a probe sequence $p$ with $N_{p}$ samples against a gallery sequence $g$ with $N_{g}$ samples, the approach in [4] is adopted, which proposed that the distance calculation process should be symmetric with respect to probe and gallery sequences. If the probe and gallery sequences were interchanged, the computed distance would be identical. The details are described as follows: each probe sample feature $\mathbf{y}_{n_{p}}$ is matched against the gallery sequence to obtain $S\left(\mathbf{y}_{n_{p}}, g\right)$ and each gallery sample feature $\mathbf{y}_{n_{g}}$ is matched against the probe sequence to obtain $S\left(\mathbf{y}_{n_{g}}, p\right)$. The matching score between the probe sequence $p$ and the gallery sequence $g$ is the sum of the mean matching score of $p$ against $g$ and that of $g$ against $p$ : $S(p, g)=\frac{1}{N_{p}} \sum_{n_{p}=1}^{N_{p}} S\left(\mathbf{y}_{n_{p}}, g\right)+\frac{1}{N_{g}} \sum_{n_{g}=1}^{N_{g}} S\left(\mathbf{y}_{n_{g}}, p\right)$. The identity of the gallery sequence with the highest matching score $S(p, g)$ is assigned to the probe sequence $p$.

Feature matching in the ETGLDA approach is similar to that in the ETG approach, with $y$ replaced by $\mathbf{z}$ in the description above. The identification performance is measured by the Cumulative Match Characteristic (CMC) [7], which plots identification rates $P_{I}$ within a given rank $k$ (rank $k$ results report the percentage of probe subjects whose true match in the gallery set was in the top $k$ matches).

4) Effects of centering on recognition: To emphasize the fact that centering is an important preprocessing in classification/recognition applications, a simple example is shown here. The proposed ETG approach is applied with and without centering of the input gait data. In both cases, the dimensionality in each mode is reduced to half of the original: $P_{1}=64, P_{2}=44, P_{3}=10$. The rank 1 and rank 5 gait recognition results using the angle distance measure and the modified angle distance (MAD) measure are shown in Fig. 12, with the number of EigenTensorGaits used $\left(H_{\mathbf{y}}\right)$ ranging from 20 to 680 . Results on centered data are significantly better than the results on the data without centering, implying that variation captured with respect to the data center is more powerful in discriminating classes than variation captured with respect to the origin.

5) Algorithm comparison: As this paper focuses on feature extraction, different feature extraction methods need to be compared first, with the other algorithmic components fixed, as shown in Table III. The following features used in the state-of-the-art gait recognition algorithms are compared: silhouettes, features extracted by performing PCA+LDA on averaged silhouettes, and features from the proposed ETGLDA approach. Other heuristic features, such as widths of the outer contour [6] and angular transforms [4] have been shown to be poorer than silhouettes so they are not compared here. As shown in Table 


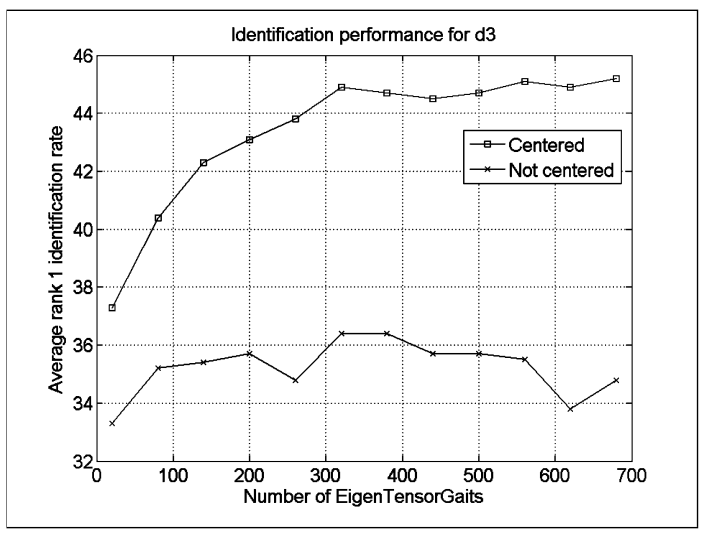

(a) Rank 1 results with angle distance.

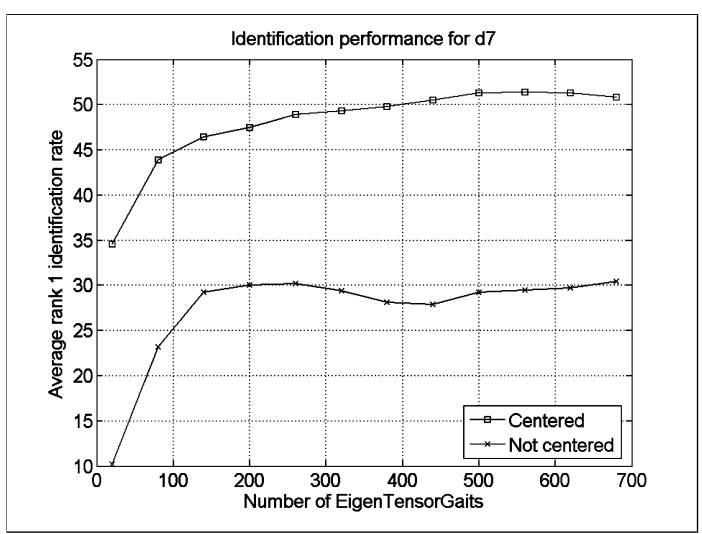

(c) Rank 1 results with MAD distance.

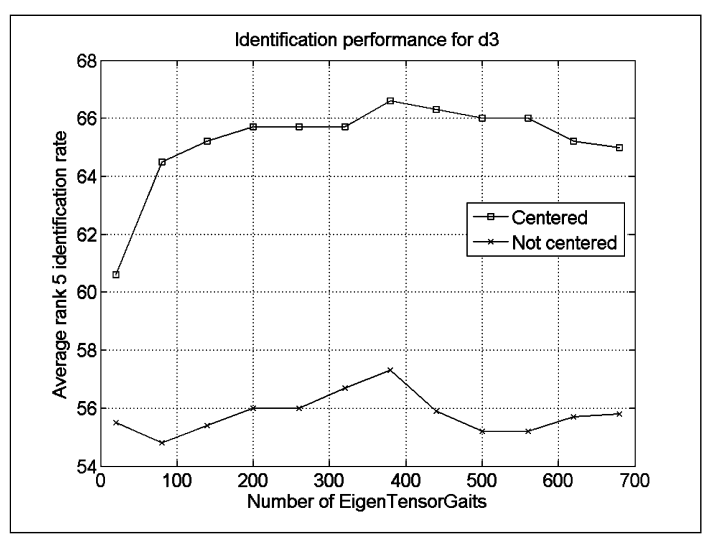

(b) Rank 5 results with angle distance.

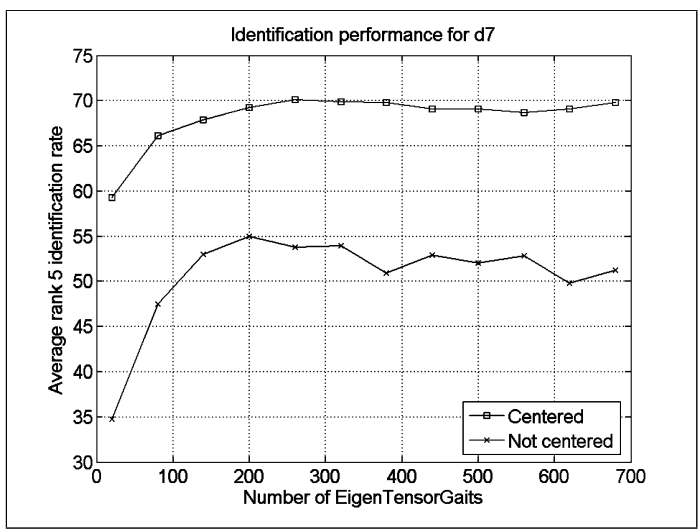

(d) Rank 5 results with MAD distance.

Fig. 12. Gait recognition results with and without centering.

III, starting with the USF silhouettes, half cycles are partitioned by the method described in Sec. V-B2 and linear interpolation is used for time-mode normalization. The frame image size is resized to $64 \times 44$ for all the feature extraction algorithms in this comparison and the matching algorithm described in Sec. V-B3 is used with three distance measures: L1, L2 and angle.

Next, the proposed ETG and ETGLDA algorithms are compared against the state-of-the-art gait recognition algorithms, with different processing steps, which is expected to have an impact on the recognition results. Algorithms that under-perform, i.e., with average rank 1 identification rate $\left(\bar{P}_{I}^{1}\right)$ less than 50\%, are not included, with the exception of the baseline algorithm [7]. Those included, as summarized in Table IV in their original formulations, are the baseline algorithm [7], the Hidden Markov Model (HMM) framework [6], the linear time normalization (LTN) algorithm [4] and the Gait Energy Image (GEI) algorithm [5]. For the HMM algorithm, the direct approach, which uses the entire silhouette 
TABLE III

COMPARISON OF FEATURE EXTRACTION METHODS FOR GAIT RECOGNITION.

\begin{tabular}{|c|c|c|c|c|}
\hline Approach & Preprocessing & Cycle partition & Feature extraction & Matching \\
\hline Baseline [7] & \multirow{5}{*}{$\begin{array}{l}\text { Baseline (half } \\
\text { image size) } \\
\text { and time-mode } \\
\text { linear } \\
\text { interpolation }\end{array}$} & \multirow{5}{*}{$\begin{array}{l}\text { Running } \\
\text { average } \\
\text { filtering } \\
\text { and minima } \\
\text { detection }\end{array}$} & \multirow{3}{*}{ Silhouettes } & \multirow{5}{*}{$\begin{array}{c}\text { L1, L2, } \\
\text { or angle } \\
\text { with } \\
\text { symmetric } \\
\text { matching }\end{array}$} \\
\hline HMM [6] & & & & \\
\hline LTN [4] & & & & \\
\hline GEI [5] & & & PCA+LDA on averaged silhouette & \\
\hline ETGLDA & & & MPCA+LDA on gait tensor sample & \\
\hline
\end{tabular}

as the feature, is chosen for comparison since the indirect approach, which uses the width of the outer contour of the silhouette as the feature, has $\bar{P}_{I}^{1}=46 \%$. For the LTN algorithm, the LTN using the silhouette feature (LTN-S) is chosen for comparison since the LTN using the angular feature (LTN-A) has $\bar{P}_{I}^{1}=41 \%$. For the GEI method, the results involving the synthetic templates are not included for fair comparison. Table IV illustrates the differences between algorithms. It should be pointed out that in the HMM approach, besides feature extraction and matching, HMM parameter estimation (training) is a major component too and it is not shown in the table.

In addition, there is a recent work applying the multilinear LDA algorithm in [30], [31] to the problem of gait recognition using matrix representation (the average of silhouettes) [32], which can be viewed as a second-order tensor representation where the time-mode information is ignored. This is fundamentally different from the natural third-order tensor representation used in the MPCA-based approaches. As mentioned before, the algorithm in [30] does not converge, as shown in Fig. 13, and their results are shown to be very sensitive to parameter settings, as shown in Fig. 4 of [32]. Furthermore, exhaustive testing is used to determine the number of iterations and the subspace dimension in [32], which is not practical due to its high computational cost. The authors did their best to faithfully implement the gait recognition algorithm in [32] based on [30] and tested 2125 parameter configurations, evenly sampled from the total 56320 possible parameter configurations. The best result, obtained after five iterations with projected dimensions of $30 \times 5$, has (average) rank 1 and rank 5 recognition rates of $42 \%$ and $57 \%$, respectively, on the gait data sets used in this paper. These results are poorer than the recognition rates obtained by the baseline algorithm. Hence, the methods in [32] or [30] are not included in the state-of-the-art gait recognition set used for comparison purposes.

6) Gait recognition results and discussions: In the gait recognition experiments, the number of eigenvectors kept in each mode is: $P_{1}=61, P_{2}=42$ and $P_{3}=17$, and they are determined by setting 


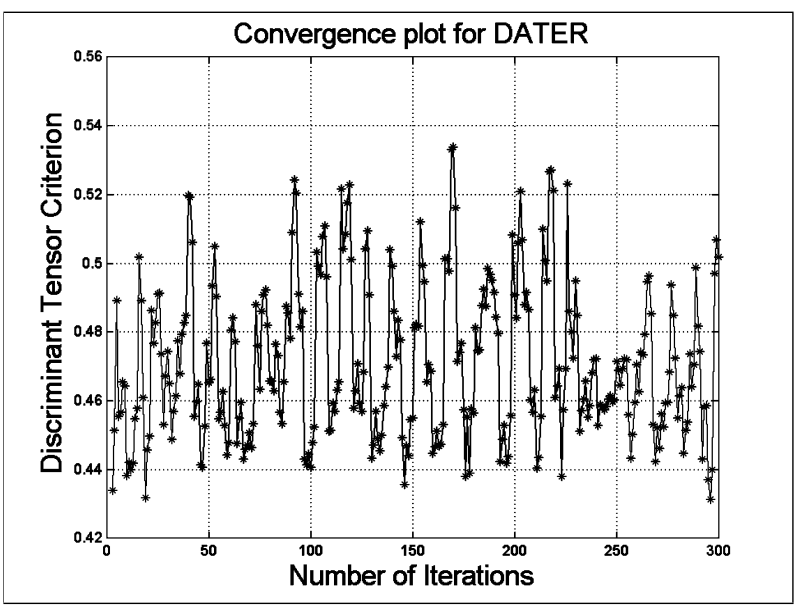

Fig. 13. The evolution of the objective criterion over iterations when the DATER algorithm in [30] is applied on the gait data.

TABLE IV

COMPARISON OF THE STATE-OF-THE-ART GAIT RECOGNITION ALGORITHMS.

\begin{tabular}{|c|c|c|c|c|}
\hline Approach & Preprocessing & Cycle partition & Feature extraction & Matching \\
\hline Baseline [7] & Baseline & $\begin{array}{l}\text { Period estimation from median } \\
\text { of distances between minima }\end{array}$ & Silhouettes & $\begin{array}{l}\text { Spatial-temporal } \\
\text { correlation }\end{array}$ \\
\hline HMM [6] & Baseline & Adaptive filter & Silhouettes & Viterbi algorithm \\
\hline LTN [4] & $\begin{array}{c}\text { Baseline \& silhouette } \\
\text { refinement }\end{array}$ & $\begin{array}{l}\text { Autocorrelation, optimal } \\
\text { filter, merging }\end{array}$ & Silhouettes & $\begin{array}{l}\text { LTN distance with } \\
\text { symmetric matching }\end{array}$ \\
\hline GEI [5] & Baseline & $\begin{array}{l}\text { Maximum entropy } \\
\text { spectrum estimation }\end{array}$ & $\begin{array}{c}\text { PCA+LDA on } \\
\text { averaged silhouette }\end{array}$ & $\begin{array}{l}\text { minimum Euclidean } \\
\text { distance to class mean }\end{array}$ \\
\hline ETG & \multirow{2}{*}{$\begin{array}{l}\text { Baseline \& time-mode } \\
\text { linear interpolation }\end{array}$} & \multirow{2}{*}{$\begin{array}{l}\text { Running average } \\
\text { filter }\end{array}$} & EigenTensorGaits & \multirow{2}{*}{$\begin{array}{l}\text { Nearest neighbor with } \\
\text { symmetric matching }\end{array}$} \\
\hline ETGLDA & & & ETG+LDA & \\
\hline
\end{tabular}

$Q^{(1)}=Q^{(2)}=Q^{(3)}=Q=0.97$, which captures approximately $92 \%$ of the total energy variation of the gallery gait samples in the projected tensor subspace. Although with the same $Q$, similar amount of variation is kept in each mode, the amount of dimensionality reduction in the image spatial modes ( $52 \%$ for the column mode and $48 \%$ for the row mode) is more significant than the the-mode $(15 \%)$. This indicates that there are more redundancy in the spatial modes than in the time mode. For the ETGLDA approach, the dimension of the feature vector $\mathbf{z}$ is fixed to be the maximum that can be obtained by classical LDA: $H_{\mathbf{z}}=C-1=70$, where $C$ is the number of gallery subjects (classes).

Two key design factors are varied to study their effects and to determine the best choices for them: the number of selected EigenTensorGaits $H_{\mathbf{y}}$ and the distance measures. For different $H_{\mathbf{y}}$ (up to 800) and 
for the distance measures listed in Table I, the average rank 1 and rank 5 identification rates are plotted in Figs. 14(a) and 14(b), respectively, for the ETG approach, and in Figs. 14(c) and 14(d), respectively, for the ETGLDA approach. For the ETG approach, the modified angle distance (MAD) measure, with the proposed weight vector $\mathbf{w}$, significantly outperforms all the other distance measures for $H_{\mathbf{y}}>200$. For the ETGLDA approach, the angle and MAD measures outperform all the other measures at rank 1 and the MAD measure is better than the angle at rank 5 for $H_{\mathbf{y}}<200$. Thus, both approaches choose MAD as the distance measure. With MAD, when more and more eigentensors are included, i.e., $H_{\mathbf{y}}$ increases, the ETG identification rate keeps increasing first, except some small fluctuations, and becomes steady beyond a certain point, indicating that most of the EigenTesnorGaits selected first are good features for classification. For the ETGLDA approach, due to the LDA feature extraction process where the feature vector length is $C-1$, the performance against $H_{\mathbf{y}}$ is different: with the MAD measure, the identification rates (rank 1 and 5) reach maximum around $H_{\mathbf{y}}=200$ and drop at a higher rate for $H_{\mathbf{y}}>600$, suggesting that more EigenTensorGaits (than 200) may lower the performance of ETGLDA.

Table V compares the feature extraction algorithms in Table III. The feature vector length is $70(C-1)$ for both the GEI (PCA+LDA on averaged silhouette) and ETGLDA (MPCA+LDA on gait tensor sample) approaches. The PCA in the GEI approach results in a feature vector of length 142, as recommended by the authors of [5], and the ETGLDA results shown are obtained with $H_{\mathbf{y}}=200$, determined empirically. The table shows that both the GEI and ETGLDA features outperform the silhouettes features significantly, and the proposed ETGLDA features outperform the GEI features. Particularly, in the average rank 1 identification rate, the ETGLDA features with the (best-performing) angle distance measure result in about $18 \%$ improvement over the GEI features with the (best-performing) L1 distance measure, indicating that the proposed MPCA-based approach is more powerful in recognition than the PCA-based approach.

TABLE V

COMPARISON OF FEATURE EXTRACTION METHODS.

\begin{tabular}{|c|c|c|c|c|c|c|}
\hline \multirow{2}{*}{ Feature extraction method } & \multicolumn{3}{|c|}{$\bar{P}_{I}(\%)$ at Rank 1 with } & \multicolumn{3}{c|}{$\bar{P}_{I}(\%)$ at Rank 5 with } \\
\cline { 2 - 7 } & L1 & L2 & Angle & L1 & L2 & Angle \\
\hline \hline Silhouettes & 34 & 35 & 33 & 54 & 54 & 53 \\
\hline PCA+LDA on averaged silhouette & 45 & 44 & 43 & 68 & 69 & 69 \\
\hline MPCA+LDA on gait tensor sample & 47 & 47 & 53 & 67 & 70 & 73 \\
\hline
\end{tabular}

Based on empirical study, the best gait recognition performance for the ETG and ETGLDA approaches is obtained with $H_{\mathbf{y}}=620$ and $H_{\mathbf{y}}=170$, respectively, using MAD. The detailed results are depicted 


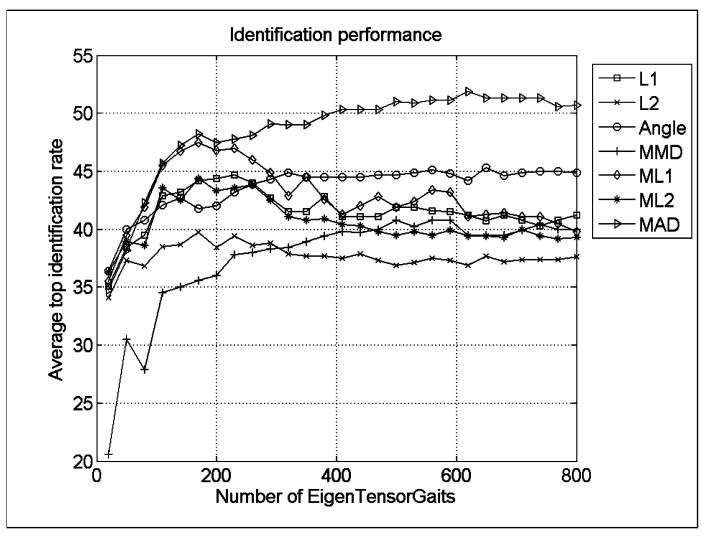

(a) Rank 1 identification performance of ETG.

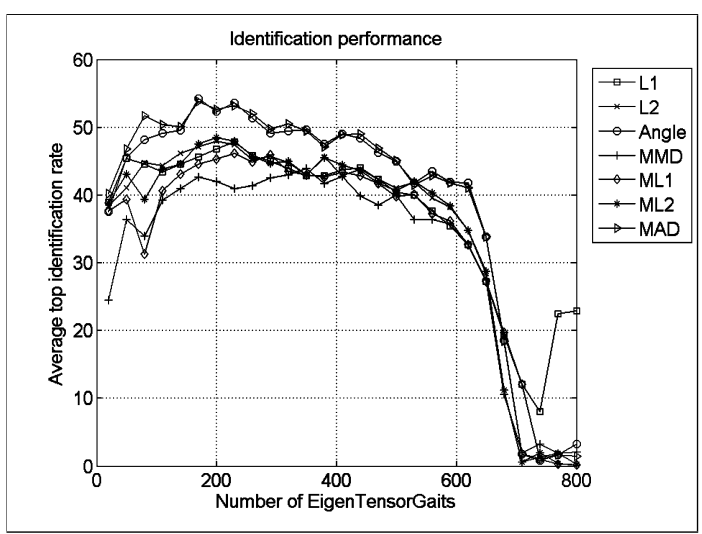

(c) Rank 1 identification performance of ETGLDA.

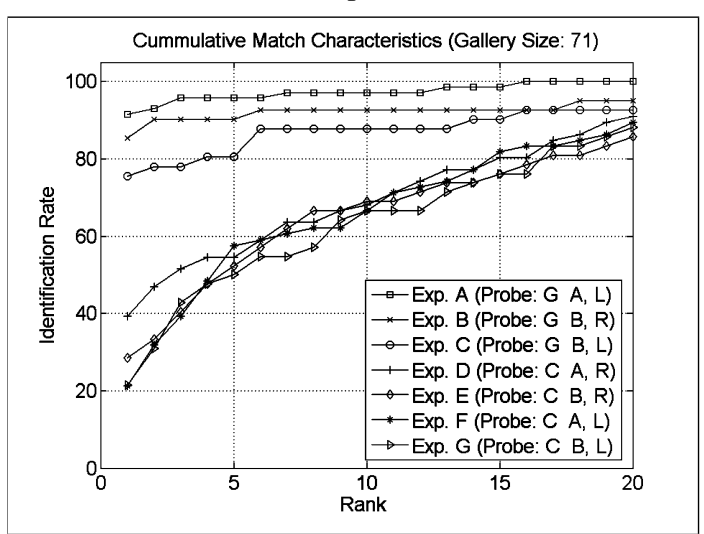

(e) CMC curves up to rank 20 for ETG.

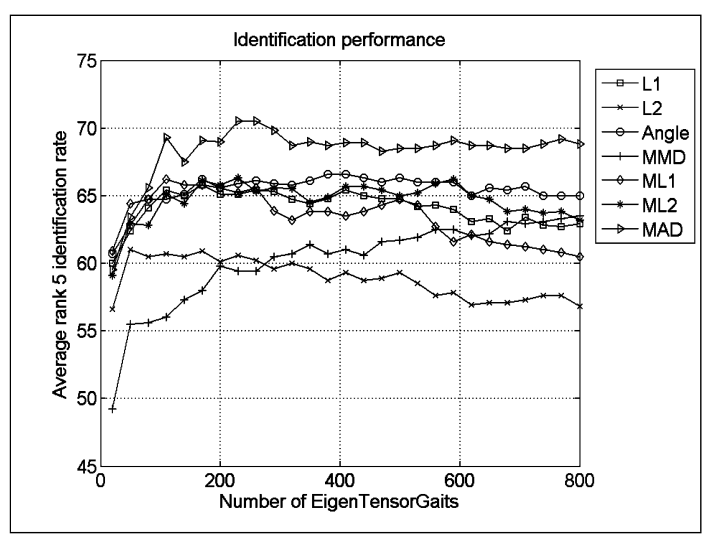

(b) Rank 5 identification performance of ETG.

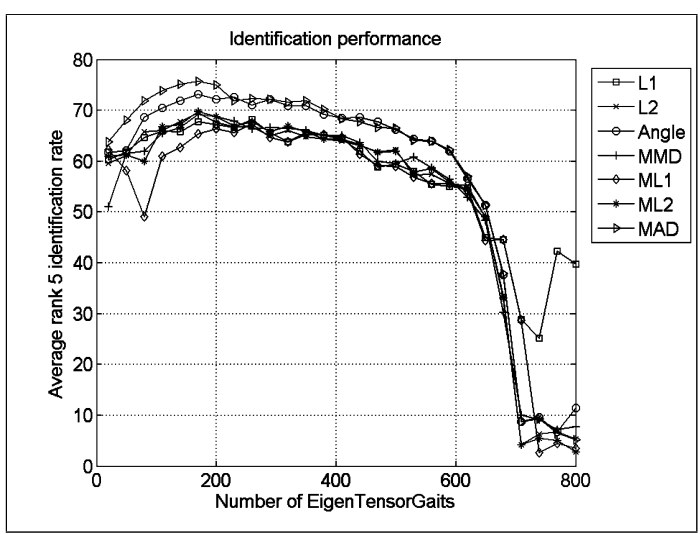

(d) Rank 5 identification performance of ETGLDA.

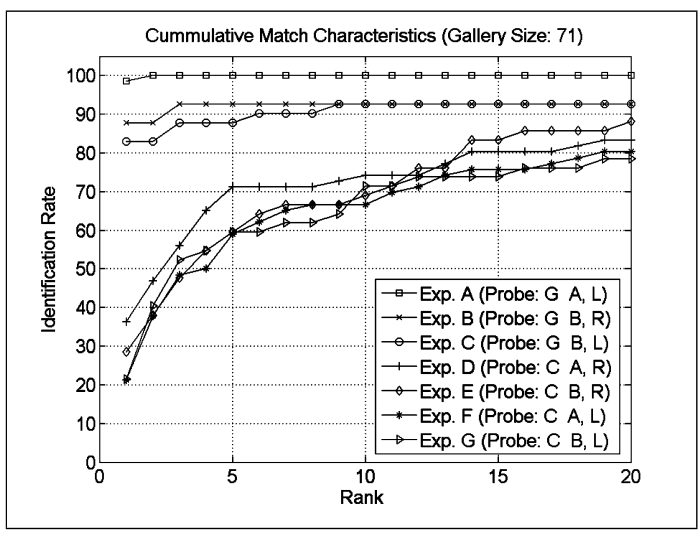

(f) CMC curves up to rank 20 for ETGLDA.

Fig. 14. Gait recognition results: dependency on $H_{\mathbf{y}}$ and the distance measure used, and the CMC curves.

using the CMCs in Figs. 14(e) and 14(f), and they are compared with the state-of-the-art gait recognition algorithms in Table VI, where the rank 1 and rank 5 identification rates are listed for each probe (A to G) together with their averages. The best results for all the probe and rank combinations are highlighted 
by boldface font in the table. From the table, the HMM, LTN, GEI, ETG and ETGLDA algorithms have no significant difference in the rank 1 performance, although LTN is slightly poorer, and they outperform the baseline results by more than $19 \%$. For the rank 5 performance, ETGLDA has the best performance.

TABLE VI

COMPARISON OF THE MPCA-BASED AND THE STATE-OF-THE-ART GAIT RECOGNITION ALGORITHMS.

\begin{tabular}{|c|c|c|c|c|c|c|c|c|c|c|c|c|}
\hline & \multicolumn{9}{|c}{$P_{I}(\%)$ at Rank } & \multicolumn{4}{c|}{$P_{I}(\%)$ at Rank } \\
\cline { 2 - 16 } Probe & Baseline & HMM & LTN & GEI & ETG & ETGLDA & Baseline & HMM & LTN & GEI & ETG & ETGLDA \\
\hline \hline A (GAL) & 79 & 99 & 94 & $\mathbf{1 0 0}$ & 92 & 99 & 96 & $\mathbf{1 0 0}$ & 99 & $\mathbf{1 0 0}$ & 96 & $\mathbf{1 0 0}$ \\
\hline B (GBR) & 66 & $\mathbf{8 9}$ & 83 & 85 & 85 & 88 & 81 & 90 & 85 & 85 & 90 & $\mathbf{9 3}$ \\
\hline C (GBL) & 56 & 78 & 78 & 80 & 76 & $\mathbf{8 3}$ & 76 & $\mathbf{9 0}$ & 83 & 88 & 81 & 88 \\
\hline D (CAR) & 29 & 35 & 33 & 30 & $\mathbf{3 9}$ & 36 & 61 & 65 & 65 & 55 & 55 & $\mathbf{7 1}$ \\
\hline E (CBR) & 24 & 29 & 24 & $\mathbf{3 3}$ & 29 & 29 & 55 & 65 & $\mathbf{6 7}$ & 55 & 52 & 60 \\
\hline F (CAL) & $\mathbf{3 0}$ & 18 & 17 & 21 & 21 & 21 & 46 & $\mathbf{6 0}$ & 58 & 41 & 58 & 59 \\
\hline G (CBL) & 10 & 24 & 21 & $\mathbf{2 9}$ & 21 & 21 & 33 & 50 & 48 & 48 & 50 & $\mathbf{6 0}$ \\
\hline \hline Average & 42 & 53 & 50 & $\mathbf{5 4}$ & 52 & $\mathbf{5 4}$ & 64 & 74 & 72 & 67 & 69 & $\mathbf{7 6}$ \\
\hline
\end{tabular}

From the comparisons in Table IV, the performance of the HMM framework is mainly contributed to the adaptive filter used for cycle partition, the Viterbi algorithm used for probabilistic matching, and the iterative training of the HMM parameters, while the performance of LTN is mainly contributed to the silhouette refinement, the robust cycle partition procedure and the LTN distance matching strategy. Besides PCA+LDA in feature extraction, the GEI algorithm utilizes a robust estimator as well for the cycle partition taking into account of the periodic nature of the gait signal and it seems that this tends to improve the recognition performance. To summarize, silhouette refinement could be a beneficial step for gait recognition and robust cycle partition seems to be an important component in these start-of-the-art gait recognition algorithms. In addition, robust matching algorithms such as the Viterbi algorithm used in HMM have great potential for gait recognition as well.

Finally, the ETGLDA approach has some small gain (about $4 \%$ improvement on average) over the ETG approach in rank 1 performance, especially on Probes A and C. In rank 5 performance, the ETGLDA approach has greater improvement (about $10 \%$ on average) over the ETG approach. Thus, the combination of the ETG approach with a classical LDA does result in better recognition results and the combination with other linear subspace algorithms are promising future directions as well. On the whole, despite a design without optimizing the preprocessing, cycle partition and matching algorithms, the MPCAbased approaches to gait recognition are highly competitive against the state-of-the-art gait recognition 
algorithms. The EigenTensorGaits, and EigenTensorObjects in general, are very promising features for tensor object recognition.

\section{CONCLUSIONS}

In this paper, a new multilinear principal component analysis (MPCA) framework is proposed for analysis of tensor objects, such as 2D/3D images and video sequences. MPCA determines a multilinear projection onto a tensor subspace of lower dimensionality that captures most of the signal variation present in the original tensorial representation. An iterative solution was formulated. Issues including initialization, convergence and subspace dimensionality determination were discussed in details. An MPCA-based tensor object recognition framework was then introduced and applied to the problem of gait recognition. Each half cycle of a gait silhouette sequence was treated as a gait sample and the proposed framework was used to devise a novel gait representation called EigenTensorGait. Comparisons with the start-of-the-art algorithms indicated that the MPCA-based approach is a very promising tool for gait recognition and its performance can be further improved by silhouette refinement, robust cycle partition and matching algorithms.

\section{ACKNOWLEDGMENTS}

The authors would like to thank the anonymous reviewers for their insightful and constructive comments. The authors would also like to thank Prof. Sarkar from the University of South Florida for providing the Gait Challenge data sets. This work is partially supported by the Ontario Centres of Excellence through the Communications and Information Technology Ontario Partnership Program and the Bell University Labs - at the University of Toronto.

\section{APPENDIX A}

\section{LIST OF SYMBOLS AND PROOFS}

\section{A. List of symbols}

Table VII summarizes the list of fundamental symbols defined in this paper for quick reference.

\section{B. Proof of Theorem 1}

Proof: The mean tensor of all the projected samples is $\overline{\mathcal{Y}}=\frac{1}{M} \sum_{m=1}^{M} \mathcal{Y}_{m}=\overline{\mathcal{X}} \times{ }_{1} \tilde{\mathbf{U}}^{(1)^{T}} \times_{2}$ $\tilde{\mathbf{U}}^{(2)^{T}} \ldots \times{ }_{N} \tilde{\mathbf{U}}^{(N)^{T}}, m=1, \ldots, M$. 
TABLE VII

LIST OF SYMBOLS.

\begin{tabular}{ll}
\hline $\mathcal{X}_{m}$ & the $m^{t h}$ input tensor sample, $m=1, \ldots, M$ \\
$\tilde{\mathbf{U}}^{(n)}$ & the $n^{t h}$ projection matrix, $n=1, \ldots, N$ \\
$\mathcal{Y}_{m}$ & the projection of $\mathcal{X}_{m}$ on $\left\{\tilde{\mathbf{U}}^{(n)}\right\}$ \\
$\Psi_{\mathcal{Y}}$ & the total tensor scatter of $\left\{\mathcal{Y}_{m}, m=1, \ldots, M\right\}$ \\
$\boldsymbol{\Phi}^{(n)}$ & the $n$-mode matrix defined in (5) \\
$\tilde{\mathbf{U}}_{\boldsymbol{\Phi}}^{(n)}$ & the kroneker product defined in (6) \\
$\tilde{\mathcal{X}}_{m}, \tilde{\mathcal{Y}}_{m}$ & the centered versions of $\mathcal{X}_{m}, \mathcal{Y}_{m}$ \\
$\boldsymbol{\Phi}^{(n) *}, \mathbf{U}^{(n) *}$ & $\boldsymbol{\Phi}^{(n)}$ and $\tilde{\mathbf{U}}^{(n)}$ for full projection \\
$\mathcal{Y}_{v a r}^{*}$ & the total scatter tensor for full projection \\
$\lambda_{i_{n}}^{(n) *}$ & the $i_{n}^{t h} n$-mode eigenvalue for full projection \\
$\Psi_{\mathcal{Y}_{0}}$ & the total tensor scatter for the FPT initialization \\
$\Psi_{\mathcal{X}}$ & the input total tensor scatter \\
$\tilde{\mathcal{Y}}_{v a r}^{*}, \hat{\mathcal{Y}}_{v a r}^{*}$ & truncated versions of $\mathcal{Y}_{v a r}^{*}$ \\
$k$ & the iteration step index in the MPCA algorithm \\
$t$ & the SMT step index \\
$\delta_{t}^{(n)}$ & the $p_{n}^{t h} n$-mode eigenvalue corresponding to $\tilde{\mathbf{U}}^{(n)}$ \\
$\tilde{\lambda}_{P_{n}}^{(n)}$ & the scatter loss rate defined in (11) \\
$\mathbf{y}_{m}$ & the $P_{n}^{\text {th }} n$-mode eigenvalue of the reconstructed \\
$\mathbf{z}_{m}$ & input tensors
\end{tabular}

Write the objective function (4) in terms of the input tensor samples as:

$$
\Psi_{\mathcal{Y}}=\sum_{m=1}^{M}\left\|\mathcal{Y}_{m}-\overline{\mathcal{Y}}\right\|_{F}^{2}=\sum_{m=1}^{M}\left\|\left(\mathcal{X}_{m}-\overline{\mathcal{X}}\right) \times_{1} \tilde{\mathbf{U}}^{(1)^{T}} \times_{2} \tilde{\mathbf{U}}^{(2)^{T}} \ldots \times_{N} \tilde{\mathbf{U}}^{(N)^{T}}\right\|_{F}^{2} .
$$

From the definition of the Frobenius norm for a tensor and that for a matrix, $\|\mathcal{A}\|_{F}=\left\|\mathbf{A}_{(n)}\right\|_{F}$, and from (2), $\Psi_{\mathcal{Y}}$ can be expressed using the equivalent matrix representation by $n$-mode unfolding as follows:

$$
\Psi_{\mathcal{Y}}=\sum_{m=1}^{M}\left\|\mathbf{Y}_{m(n)}-\overline{\mathbf{Y}}_{(n)}\right\|_{F}^{2}=\sum_{m=1}^{M}\left\|\tilde{\mathbf{U}}^{(n)^{T}} \cdot\left(\mathbf{X}_{m(n)}-\overline{\mathbf{X}}_{(n)}\right) \cdot \tilde{\mathbf{U}}_{\Phi^{(n)}}\right\|_{F}^{2},
$$

Since $\|\mathbf{A}\|_{F}^{2}=\operatorname{trace}\left(\mathbf{A} \mathbf{A}^{T}\right), \Psi_{\mathcal{Y}}$ can be written in terms of the $n$-mode total scatter matrix of the 
projected tensor samples:

$$
\begin{aligned}
\Psi_{\mathcal{Y}} & =\sum_{m=1}^{M} \operatorname{trace}\left(\tilde{\mathbf{U}}^{(n)^{T}} \cdot\left(\mathbf{X}_{m(n)}-\overline{\mathbf{X}}_{(n)}\right) \cdot \tilde{\mathbf{U}}_{\Phi^{(n)}} \cdot \tilde{\mathbf{U}}_{\Phi^{(n)}}^{T} \cdot\left(\mathbf{X}_{m(n)}-\overline{\mathbf{X}}_{(n)}\right)^{T} \cdot \tilde{\mathbf{U}}^{(n)}\right) \\
& =\operatorname{trace}\left(\tilde{\mathbf{U}}^{(n)^{T}} \cdot \boldsymbol{\Phi}^{(n)} \cdot \tilde{\mathbf{U}}^{(n)}\right),
\end{aligned}
$$

Therefore, for given $\tilde{\mathbf{U}}^{(1)}, \ldots, \tilde{\mathbf{U}}^{(n-1)}, \tilde{\mathbf{U}}^{(n+1)}, \ldots, \tilde{\mathbf{U}}^{(N)}$, the total scatter $\Psi_{\mathcal{Y}}$ is maximized if and only if $\operatorname{trace}\left(\tilde{\mathbf{U}}^{(n)^{T}} \cdot \boldsymbol{\Phi}^{(n)} \cdot \tilde{\mathbf{U}}^{(n)}\right)$ is maximized. The maximum of trace $\left(\tilde{\mathbf{U}}^{(n)^{T}} \cdot \boldsymbol{\Phi}^{(n)} \cdot \tilde{\mathbf{U}}^{(n)}\right)$ is obtained if $\tilde{\mathbf{U}}^{(n)}$ consists of the $P_{n}$ eigenvectors of the matrix $\boldsymbol{\Phi}^{(n)}$ corresponding to the largest $P_{n}$ eigenvalues.

\section{Proof of Lemma 4}

Lemma 4: When $P_{n}=I_{n}$ for $n=1, \ldots, N, \tilde{\mathbf{U}}_{\Phi^{(n)}} \cdot \tilde{\mathbf{U}}_{\Phi^{(n)}}^{T}$ is an identity matrix.

Proof: By successive application of the transpose property of the Kronecker product $(\mathbf{A} \otimes \mathbf{B})^{T}=$ $\mathbf{A}^{T} \otimes \mathbf{B}^{T}[38]:$

$$
\tilde{\mathbf{U}}_{\Phi^{(n)}}^{T}=\left(\tilde{\mathbf{U}}^{(n+1)^{T}} \otimes \tilde{\mathbf{U}}^{(n+2)^{T}} \otimes \ldots \otimes \tilde{\mathbf{U}}^{(N)^{T}} \otimes \tilde{\mathbf{U}}^{(1)^{T}} \otimes \tilde{\mathbf{U}}^{(2)^{T}} \otimes \ldots \tilde{\mathbf{U}}^{(n-1)^{T}}\right) .
$$

By the Kronecker product theorem $(\mathbf{A} \otimes \mathbf{B})(\mathbf{C} \otimes \mathbf{D})=(\mathbf{A C} \otimes \mathbf{B D})[38]$,

$$
\tilde{\mathbf{U}}_{\Phi(n)} \cdot \tilde{\mathbf{U}}_{\Phi^{(n)}}^{T}=\left(\tilde{\mathbf{U}}^{(n+1)} \tilde{\mathbf{U}}^{(n+1)^{T}} \otimes \ldots \otimes \tilde{\mathbf{U}}^{(N)} \tilde{\mathbf{U}}^{(N)^{T}} \otimes \tilde{\mathbf{U}}^{(1)} \tilde{\mathbf{U}}^{(1)^{T}} \otimes \ldots \tilde{\mathbf{U}}^{(n-1)} \tilde{\mathbf{U}}^{(n-1)^{T}}\right) .
$$

For all $n$, when $P_{n}=I_{n}, \tilde{\mathbf{U}}^{(n)}$ is a square matrix and $\tilde{\mathbf{U}}^{(n)^{T}} \tilde{\mathbf{U}}^{(n)}=\mathbf{I}_{I_{n}}$, where $\mathbf{I}_{I_{n}}$ is an $I_{n} \times I_{n}$ identity matrix. Then, $\tilde{\mathbf{U}}^{(n)^{-1}}=\tilde{\mathbf{U}}^{(n)^{T}}$ and $\tilde{\mathbf{U}}^{(n)} \tilde{\mathbf{U}}^{(n)^{T}}=\mathbf{I}_{I_{n}}$. Thus, $\tilde{\mathbf{U}}_{\Phi^{(n)}} \cdot \tilde{\mathbf{U}}_{\boldsymbol{\Phi}^{(n)}}^{T}=\mathbf{I}_{I_{1} \times I_{2} \times \ldots \times I_{n-1} \times I_{n+1} \times \ldots \times I_{N}}$.

\section{Proof of Theorem 2}

The following lemma explains the relationship between the eigenvalues of two covariance matrices that are closely related.

Lemma 5: Let $\mathbf{X} \in \mathbb{R}^{I_{1} \times I_{2}}$ be a sample matrix with $I_{2}$ samples. $\tilde{\mathbf{X}} \in \mathbb{R}^{I_{1} \times P_{2}}$ contains $P_{2}<I_{2}$ samples from $\mathbf{X}$, and $\mathbf{E} \in \mathbb{R}^{I_{1} \times\left(I_{2}-P_{2}\right)}$ contains the rest. Let $\lambda_{i_{1}}$ and $\tilde{\lambda}_{i_{1}}$, denote the $i_{1}^{\text {th }}$ eigenvalues of $\mathbf{X X}^{T}$ and $\tilde{\mathbf{X}} \tilde{\mathbf{X}}^{T}$, respectively. Then, $\tilde{\lambda}_{i_{1}} \leq \lambda_{i_{1}}$, for $i_{1}=1, \ldots, I_{1}$.

Proof: Without loss of generality, let $\tilde{\mathbf{X}}=\mathbf{X}\left(:, 1: P_{2}\right)$ and $\mathbf{E}=\mathbf{X}\left(:,\left(P_{2}+1\right): I_{2}\right) . \mathbf{X X}^{T}$ and $\tilde{\mathbf{X}} \tilde{\mathbf{X}}{ }^{T}$ are both sample covariance matrices and hence symmetric. $\mathbf{E}$ is related to them by $\mathbf{X} \mathbf{X}^{T}=\tilde{\mathbf{X}} \tilde{\mathbf{X}}^{T}+\mathbf{E E}^{T}$. $\mathbf{E E}^{T}$ is a covariance matrix as well and hence it is positive semidefinite. From the Weyl's theorem [35], 
which is derived from the Courant-Fisher "min-max theorem" [35], the eigenvalues of $\tilde{\mathbf{X}} \tilde{\mathbf{X}}{ }^{T}$ is not greater than the corresponding eigenvalues of $\tilde{\mathbf{X}} \tilde{\mathbf{X}}^{T}+\mathbf{E E}^{T}=\mathbf{X} \mathbf{X}^{T}$, i.e., $\tilde{\lambda}_{i_{1}} \leq \lambda_{i_{1}}$, for $i_{1}=1, \ldots, I_{1}$.

The proof of Theorem 2 follows:

Proof: For $n=1, \check{\mathbf{X}}_{m(1)}=\check{\mathbf{U}}^{(1)} \cdot \check{\mathbf{Y}}_{m(1)} \cdot \mathbf{U}_{\Phi^{(1) *}}^{T}, m=1, \ldots, M$. Thus,

$$
\begin{aligned}
\check{\mathbf{\Phi}}^{(1)} & =\sum_{m} \check{\mathbf{X}}_{m(1)} \mathbf{U}_{\boldsymbol{\Phi}^{(1) *}} \mathbf{U}_{\Phi^{(1) *}}^{T} \check{\mathbf{X}}_{m(1)}^{T} \\
& =\sum_{m} \check{\mathbf{U}}^{(1)} \cdot \check{\mathbf{Y}}_{m(1)} \cdot \mathbf{U}_{\Phi^{(1) *}}^{T} \mathbf{U}_{\Phi^{(1) *}} \cdot \mathbf{U}_{\Phi^{(1) *}}^{T} \mathbf{U}_{\Phi^{(1) *}} \cdot \check{\mathbf{Y}}_{m(1)}^{T} \cdot \check{\mathbf{U}}^{(1)^{T}} \\
& =\sum_{m} \check{\mathbf{U}}^{(1)} \cdot \check{\mathbf{Y}}_{m(1)} \check{\mathbf{Y}}_{m(1)}^{T} \cdot \check{\mathbf{U}}^{(1)^{T}}=\check{\mathbf{U}}^{(1)} \cdot \sum_{m}\left(\check{\mathbf{Y}}_{m(1)} \check{\mathbf{Y}}_{m(1)}^{T}\right) \cdot \check{\mathbf{U}}^{(1)^{T}}
\end{aligned}
$$

where $\mathbf{U}_{\boldsymbol{\Phi}^{(1) *}}^{T} \mathbf{U}_{\boldsymbol{\Phi}^{(1) *}}$ results in an identity matrix. Since $\check{\mathbf{Y}}_{m(1)}$ is simply the first $P_{1}$ rows of $\tilde{\mathbf{Y}}_{m(1)}$, $\hat{\lambda}_{i_{1}}^{(1)}=\lambda_{i_{1}}^{(1) *}$ for $i_{1}=1, \ldots, P_{1}$, and $\hat{\lambda}_{i_{1}}^{(1)}=0$ for $i_{1}=P_{1}+1, \ldots, I_{1}$.

Similarly for $n \neq 1, \check{\mathbf{\Phi}}^{(n)}=\check{\mathbf{U}}^{(n)} \cdot \sum_{m}\left(\check{\mathbf{Y}}_{m(n)} \check{\mathbf{Y}}_{m(n)}^{T}\right) \cdot \check{\mathbf{U}}^{(n)^{T}}$. The columns of $\check{\mathbf{Y}}_{m(n)}$ are a subset of the columns of $\tilde{\mathbf{Y}}_{m(n)}$. Therefore, by Lemma $5, \hat{\lambda}_{i_{n}}^{(n)} \leq \lambda_{i_{n}}^{(n) *}$. Since $\sum_{i_{1}} \hat{\lambda}_{i_{1}}^{(1)}<\sum_{i_{1}} \lambda_{i_{1}}^{(1) *}, \sum_{i_{n}} \hat{\lambda}_{i_{n}}^{(n)}=$ $\sum_{i_{1}} \hat{\lambda}_{i_{1}}^{(1)}<\sum_{i_{n}} \lambda_{i_{n}}^{(n) *}=\sum_{i_{1}} \lambda_{i_{1}}^{(1) *}$. Thus, for each mode, at least for one value of $i_{n}, \hat{\lambda}_{i_{n}}^{(n)}<\lambda_{i_{n}}^{(n) *}$.

\section{E. Proof of Theorem 3}

Proof: For the lower bound, considering the 1-mode eigenvalues $\lambda_{i_{1}}^{(1) *}$ first,

$$
\begin{aligned}
\Psi_{\mathcal{X}}-\Psi_{\mathcal{Y}_{0}} & =\sum_{i_{1}=1}^{I_{1}} \sum_{i_{2}=1}^{I_{2}} \ldots \sum_{i_{N}=1}^{I_{N}} \mathcal{Y}_{\text {var }}^{*}\left(i_{1}, i_{2}, \ldots, i_{N}\right)-\sum_{i_{1}=1}^{P_{1}} \sum_{i_{2}=1}^{P_{2}} \ldots \sum_{i_{N}=1}^{P_{N}} \mathcal{Y}_{v a r}^{*}\left(i_{1}, i_{2}, \ldots, i_{N}\right) \\
& \geq \sum_{i_{1}=1}^{I_{1}} \sum_{i_{2}=1}^{I_{2}} \ldots \sum_{i_{N}=1}^{I_{N}} \mathcal{Y}_{\text {var }}^{*}\left(i_{1}, i_{2}, \ldots, i_{N}\right)-\sum_{i_{1}=1}^{P_{1}} \sum_{i_{2}=1}^{I_{2}} \ldots \sum_{i_{N}=1}^{I_{N}} \mathcal{Y}_{v a r}^{*}\left(i_{1}, i_{2}, \ldots, i_{N}\right) \\
& =\sum_{i_{1}=1}^{I_{1}} \lambda_{i_{1}}^{(1) *}-\sum_{i_{1}=1}^{P_{1}} \lambda_{i_{1}}^{(1) *}=\sum_{i_{1}=P_{1}+1}^{I_{1}} \lambda_{i_{1}}^{(1) *}
\end{aligned}
$$

where $\mathcal{Y}_{v a r}^{*}$ is the total scatter tensor (corresponding to the full projection) defined in (7). The above inequality can be similarly derived for the other $n$-mode eigenvalues $\lambda_{i_{n}}^{(n) *}, \Psi_{\mathcal{X}}-\Psi_{\mathcal{Y}_{0}} \geq \sum_{i_{n}=P_{n}+1}^{I_{n}} \lambda_{i_{n}}^{(n) *}$ for $n=2, \ldots, N$. Therefore,

$$
\Psi_{\mathcal{X}}-\Psi_{\mathcal{Y}_{0}} \geq \max _{n} \sum_{i_{n}=P_{n}+1}^{I_{n}} \lambda_{i_{n}}^{(n) *}
$$


For the upper bound,

$$
\begin{aligned}
\Psi_{\mathcal{X}}-\Psi_{\mathcal{Y}_{0}}= & \sum_{i_{1}=1}^{I_{1}} \sum_{i_{2}=1}^{I_{2}} \ldots \sum_{i_{N}=1}^{I_{N}} \mathcal{Y}_{v a r}^{*}\left(i_{1}, i_{2}, \ldots, i_{N}\right)-\sum_{i_{1}=1}^{P_{1}} \sum_{i_{2}=1}^{P_{2}} \ldots \sum_{i_{N}=1}^{P_{N}} \mathcal{Y}_{v a r}^{*}\left(i_{1}, i_{2}, \ldots, i_{N}\right) \\
\leq & \sum_{i_{1}=1}^{I_{1}} \sum_{i_{2}=1}^{I_{2}} \ldots \sum_{i_{N}=1}^{I_{N}} \mathcal{Y}_{v a r}^{*}\left(i_{1}, i_{2}, \ldots, i_{N}\right)-\sum_{i_{1}=1}^{P_{1}} \sum_{i_{2}=1}^{I_{2}} \ldots \sum_{i_{N}=1}^{I_{N}} \mathcal{Y}_{v a r}^{*}\left(i_{1}, i_{2}, \ldots, i_{N}\right) \\
& +\sum_{i_{1}=1}^{I_{1}} \sum_{i_{2}=1}^{I_{2}} \ldots \sum_{i_{N}=1}^{I_{N}} \mathcal{Y}_{v a r}^{*}\left(i_{1}, i_{2}, \ldots, i_{N}\right)-\sum_{i_{1}=1}^{I_{1}} \sum_{i_{2}=1}^{P_{2}} \ldots \sum_{i_{N}=1}^{I_{N}} \mathcal{Y}_{v a r}^{*}\left(i_{1}, i_{2}, \ldots, i_{N}\right) \\
& +\ldots \\
& +\sum_{i_{1}=1}^{I_{1}} \sum_{i_{2}=1}^{I_{2}} \ldots \sum_{i_{N}=1}^{I_{N}} \mathcal{Y}_{v a r}^{*}\left(i_{1}, i_{2}, \ldots, i_{N}\right)-\sum_{i_{1}=1}^{I_{1}} \sum_{i_{2}=1}^{I_{2}} \ldots \sum_{i_{N}=1}^{P_{N}} \mathcal{Y}_{v a r}^{*}\left(i_{1}, i_{2}, \ldots, i_{N}\right) \\
= & \sum_{n=1}^{N} \sum_{i_{n}=P_{n}+1}^{I_{n}} \lambda_{i_{n}}^{(n) *} .
\end{aligned}
$$

\section{REFERENCES}

[1] L. D. Lathauwer, B. D. Moor, and J. Vandewalle, "On the best rank-1 and rank- $\left(R_{1}, R_{2}, \ldots, R_{N}\right)$ approximation of higherorder tensors," SIAM Journal of Matrix Analysis and Applications, vol. 21, no. 4, pp. 1324-1342, 2000.

[2] K. N. Plataniotis and A. N. Venetsanopoulos, Color Image Processing and Applications. Springer Verlag, Berlin, 2000.

[3] N. V. Boulgouris, D. Hatzinakos, and K. N. Plataniotis, "Gait recognition: a challenging signal processing technology for biometrics," IEEE Signal Process. Mag., vol. 22, no. 6, Nov. 2005.

[4] N. V. Boulgouris, K. N. Plataniotis, and D. Hatzinakos, "Gait recognition using linear time normalization," Pattern Recognition, vol. 39, no. 5, pp. 969-979, 2006.

[5] J. Han and B. Bhanu, "Individual recognition using gait energy image," IEEE Trans. Pattern Anal. Mach. Intell., vol. 28, no. 2, pp. 316-322, Feb. 2006.

[6] A. Kale, A. N. Rajagopalan, A. Sunderesan, N. Cuntoor, A. Roy-Chowdhury, V. Krueger, and R. Chellappa, "Identification of humans using gait," IEEE Trans. Image Process., vol. 13, no. 9, pp. 1163-1173, Sep. 2004.

[7] S. Sarkar, P. J. Phillips, Z. Liu, I. Robledo, P. Grother, and K. W. Bowyer, "The human ID gait challenge problem: Data sets, performance, and analysis," IEEE Trans. Pattern Anal. Mach. Intell., vol. 27, no. 2, pp. 162-177, Feb. 2005.

[8] K. W. Bowyer, K. Chang, and P. Flynn, "A survey of approaches and challenges in 3D and multi-modal 3D + 2D face recognition," Computer Vision and Image Understanding, vol. 101, no. 1, pp. 1-15, Jan. 2006.

[9] S. Z. Li, C. Zhao, X. Zhu, and Z. Lei, "3D+2D face recognition by fusion at both feature and decision levels," in Proc. IEEE Int. Workshop on Analysis and Modeling of Faces and Gestures, Oct. 2005.

[10] A. Colombo, C. Cusano, and R. Schettini, "3D face detection using curvature analysis," Pattern Recognition, vol. 39, no. 3, pp. 444-455, Mar. 2006.

[11] R. Chellappa, A. Roy-Chowdhury, and S. Zhou, Recognition of Humans and Their Activities Using Video. Morgan \& Claypool Publishers, 2005. 
[12] H. S. Sahambi and K. Khorasani, "A neural-network appearance-based 3-d object recognition using independent component analysis," IEEE Trans. Neural Netw., vol. 14, no. 1, pp. 138-149, Jan. 2003.

[13] C. Nolker and H. Ritter, "Visual recognition of continuous hand postures," IEEE Trans. Neural Netw., vol. 13, no. 4, pp. 983-994, Jul. 2002.

[14] R. D. Green and L. Guan, "Quantifying and recognizing human movement patterns from monocular video images-part ii: applications to biometrics," IEEE Trans. Circuits Syst. Video Technol., vol. 14, no. 2, pp. 191-198, Feb. 2004.

[15] E. Shechtman and Y. C. ad M. Irani, "Space-time super-resolution,” IEEE Trans. Pattern Anal. Mach. Intell., vol. 27, no. 4, pp. 531-545, Apr. 2005.

[16] G. Shakhnarovich and B. Moghaddam, "Face recognition in subspaces," in Handbook of Face Recognition, S. Z. Li and A. K. Jain, Eds. Springer-Verlag, 2004, pp. 141-168.

[17] J. Zhang, S. Z. Li, and J. Wang, “Manifold learning and applications in recognition,” in Intelligent Multimedia Processing with Soft Computing, Y. P. Tan, K. H. Yap, and L. Wang, Eds. Springer-Verlag, 2004, pp. 281-300.

[18] M. H. C. Law and A. K. Jain, "Incremental nonlinear dimensionality reduction by manifold learning," IEEE Trans. Pattern Anal. Mach. Intell., vol. 28, no. 3, pp. 377-391, Mar. 2006.

[19] I. T. Jolliffe, Principal Component Analysis, second edition. Springer Serires in Statistics, 2002.

[20] J. Ye, R. Janardan, and Q. Li, "GPCA: An efficient dimension reduction scheme for image compression and retrieval," in The Tenth ACM SIGKDD International Conference on Knowledge Discovery and Data Mining, 2004, pp. 354-363.

[21] J. Yang, D. Zhang, A. F. Frangi, and J. Yang, "Two-dimensional PCA: a new approach to appearance-based face representation and recognition," IEEE Trans. Pattern Anal. Mach. Intell., vol. 26, no. 1, pp. 131-137, Jan. 2004.

[22] J. Ye, "Generalized low rank approximations of matrices," Machine Learning, vol. 61, no. 1-3, pp. 167-191, 2005.

[23] X. He, D. Cai, and P. Niyogi, "Tensor subspace analysis," in Advances in Neural Information Processing Systems 18 (NIPS), 2005.

[24] L. D. Lathauwer, B. D. Moor, and J. Vandewalle, "A multilinear singualr value decomposition," SIAM Journal of Matrix Analysis and Applications, vol. 21, no. 4, pp. 1253-1278, 2000.

[25] L. D. Lathauwer and J. Vandewalle, "Dimensionality reduction in higher-order signal processing and rank- $\left(R_{1}, R_{2}, \ldots, R_{N}\right)$ reduction in multilinear algebra," Linear Algebra and its Applications, vol. 391, pp. 31-55, Nov. 2004.

[26] C. S. Lee and A. Elgammal, "Towards scalable view-invariant gait recognition: Multilinear analysis for gait," in Proc. Int. Conf. on Audio and Video-Based Biometric Person Authentication, Jul. 2005, pp. 395-405.

[27] M. A. O. Vasilescu and D. Terzopoulos, "Multilinear analysis of image ensembles: Tensorfaces," in Proc. 7th European Conference on Computer Vision, May 2002, pp. 447-460.

[28] H. Lu, K. N. Plataniotis, and A. N. Venetsanopoulos, "Multilinear principal component analysis of tensor objects for recognition," in Proc. Int. Conf. on Pattern Recognition, August 2006, pp. 776 - 779.

[29] D. Xu, S. Yan, L. Zhang, H.-J. Zhang, Z. Liu, and H.-Y. Shum;, "Concurrent subspaces analysis,” in Proc. IEEE Computer Society Conf. on Computer Vision and Pattern Recognition, vol. II, June 2005, pp. 203-208.

[30] S. Yan, D. Xu, Q. Yang, L. Zhang, X. Tang, and H.-J. Zhang, “Discriminant analysis with tensor representation,” in Proc. IEEE Computer Society Conf. on Computer Vision and Pattern Recognition, vol. I, June 2005, pp. 526-532.

[31] _ - "Multilinear discriminant analysis for face recognition," IEEE Trans. Image Process., vol. 16, no. 1, pp. 212-220, Jan. 2007.

[32] D. Xu, S. Yan, D. Tao, L. Zhang, X. Li, and H.-J. Zhang, "Human gait recognition with matrix representation," IEEE Trans. Circuits Syst. Video Technol., vol. 16, no. 7, pp. 896-903, Jul. 2006. 
[33] M. Turk and A. Pentland, "Eigenfaces for recognition,” Journal of Cognitive Neurosicence, vol. 3, no. 1, pp. 71-86, 1991.

[34] P. Kroonenberg and J. Leeuw, "Principal component analysis of three-mode data by means of alternating least squares algorithms," Psychometrika, vol. 45, no. 1, pp. 69-97, 1980.

[35] R. A. Horn and C. R. Johnson, Matrix Analysis. Cambridge Unverisity Press, 1985.

[36] P. N. Belhumeur, J. P. Hespanha, and D. J. Kriegman, "Eigenfaces vs. fisherfaces: Recognition using class specific linear projection,” IEEE Trans. Pattern Anal. Mach. Intell., vol. 19, no. 7, pp. 711-720, Jul. 1997.

[37] J. Wang, K. N. Plataniotis, and A. N. Venetsanopoulos, "Selecting discriminant eigenfaces for face recognition," Pattern Recognition Letters, vol. 26, no. 10, pp. 1470-1482, 2005.

[38] T. K. Moon and W. C. Stirling, Mathematical methods and Algorithms for Signal Processing. Prentice Hall, 2000.

[39] H. Moon and P. J. Phillips, "Computational and performance aspects of PCA-based face recognition algorithms," Perception, vol. 30, pp. 303-321, 2001.

[40] P. J. Phillips, H. Moon, S. A. Rizvi, and P. Rauss, "The FERET evaluation method for face recognition algorithms," IEEE Trans. Pattern Anal. Mach. Intell., vol. 22, no. 10, pp. 1090-1104, Oct. 2000.

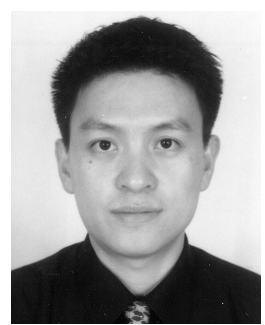

Haiping Lu (S'01) received the B.Eng. and M.Eng degrees in electrical and electronic engineering from Nanyang Technological University, Singapore, in 2001 and 2004, respectively. He is currently working towards the Ph.D. degree in the Edward S. Rogers Sr. Department of Electrical and Computer Engineering, University of Toronto, Toronto, ON, Canada.

His current research interests include statistical pattern recognition and tensor object processing, with applications in human detection, tracking and recognition.

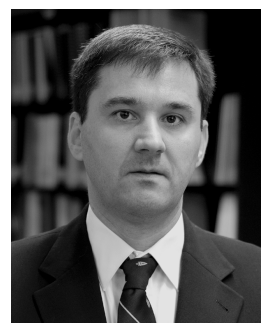

Konstantinos N. (Kostas) Plataniotis (S'90-M'92-SM'03) received his B. Eng. degree in Computer Engineering \& Informatics from University of Patras, Greece in 1988 and his M.S and Ph.D degrees in Electrical Engineering from Florida Institute of Technology(Florida Tech) in Melbourne, Florida, in 1992 and 1994 respectively. He is now an Associate Professor with The Edward S. Rogers Sr. Department of Electrical and Computer Engineering at the University of Toronto in Toronto, Ontario, Canada.

Kostas Plataniotis is a registered professional engineer in the province of Ontario, and a member of the Technical Chamber of Greece. Dr. Plataniotis is the 2005 recipient of IEEE Canada's Outstanding Engineering Educator Award "for contributions to engineering education and inspirational guidance of graduate students" and the co-recipient of the 2006 IEEE Trans. on Neural Networks Outstanding Paper Award for the published in 2003 paper " Face Recognition Using Kernel Direct Discriminant Analysis Algorithms” (Juwei Lu, Konstantinos N. Plataniotis and Anastasios N. Venetsanopoulos, IEEE TNN, Vol. 14, No. 1, 2003). 


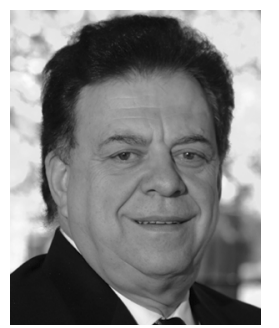

Anastasios N. Venetsanopoulos (S'66-M'69-SM'79-F'88) received the Bachelors of Electrical and Mechanical Engineering degree from the National Technical University of Athens (NTU), Greece, in 1965, and the M.S., M.Phil., and Ph.D. degrees in Electrical Engineering from Yale University in 1966, 1968 and 1969 respectively. He has served as lecturer in 138 short courses to industry and continuing education programs and as a consultant to numerous organizations; he is the co-author of four and a contributor to thirty-five books, and has published over 800 papers in refereed journals and conference proceedings. He served as the 12th Dean of the Faculty of Applied Science and Engineering of the University of Toronto and is currently the Vice President for Research and Innovation at Ryerson University, Canada.

Prof. Venetsanopoulos has served as Chair on numerous boards, councils and technical conference committees of the Institute of Electrical and Electronic Engineers (IEEE). He is a Fellow of the IEEE, a Fellow of EIC, and a Fellow of Canadian Academy of Engineering. He was awarded an Honorary Doctorate from the National Technical University of Athens, in October 1994. In October 1996 he was awarded the Excellence in Innovation Award of the Information Technology Research Centre of Ontario and Royal Bank of Canada, for innovative work in color image processing and its industrial applications. He is the recipient of the Millennium Medal of IEEE. In 2003 he was the recipient of the highest award of the Canadian IEEE, the MacNaughton Award and in 2003 he served as the Chair of the Council of Deans of Engineering of the Province of Ontario (CODE). He is the joint recipient of the 2006 IEEE Transactions on Neural Networks Outstanding Paper Award. 